\title{
Internal dynamics of Abell 2254: a merging galaxy cluster with a clumpy, diffuse radio emission
}

\author{
M. Girardi ${ }^{1,2}$, S. Bardelli ${ }^{3}$, R. Barrena ${ }^{4,5}$, W. Boschin ${ }^{6}$, F. Gastaldello ${ }^{7,8}$, and M. Nonino ${ }^{2}$ \\ 1 Dipartimento di Fisica dell'Università degli Studi di Trieste - Sezione di Astronomia, via Tiepolo 11, 34143 Trieste, Italy \\ e-mail: mgirardi@oats.inaf.it \\ 2 INAF - Osservatorio Astronomico di Trieste, via Tiepolo 11, 34143 Trieste, Italy \\ 3 INAF - Osservatorio Astronomico di Bologna, via Ranzani 1, 40127 Bologna, Italy \\ ${ }^{4}$ Instituto de Astrofísica de Canarias, C/vía Láctea s/n, 38205 La Laguna (Tenerife), Canary Islands, Spain \\ 5 Departamento de Astrofísica, Universidad de La Laguna, Av. del Astrofísico Franciso Sánchez s/n, 38205 La Laguna (Tenerife), \\ Canary Islands, Spain \\ ${ }^{6}$ Fundación Galileo Galilei - INAF, Rambla José Ana Fernández Perez 7, 38712 Breña Baja (La Palma), Canary Islands, Spain \\ 7 INAF-IASF Milano, via Bassini 15, 20133 Milano, Italy \\ 8 Department of Physics and Astronomy, University of California at Irvine, 4129, Frederick Reines Hall, Irvine, CA, \\ 92697-4575, USA
}

Received 24 May 2011 / Accepted 25 September 2011

\begin{abstract}
Context. The mechanisms giving rise to diffuse radio emission in galaxy clusters and, in particular, their connection with cluster mergers are still being debated.

Aims. We explore the internal dynamics of Abell 2254, which has been shown to host a very clumpy and irregular radio halo.

Methods. Our analysis is mainly based on redshift data for 128 galaxies acquired at the Telescopio Nazionale Galileo. We combined galaxy velocities and positions to select 110 cluster galaxies and analyze its internal dynamics. We also used new $\left(g^{\prime}, r^{\prime}, i^{\prime}\right)$ photometric data acquired at the Isaac Newton Telescope, and $\left(\mathrm{V}, i^{\prime}\right)$ photometric data available in the Subaru Archive. X-ray data from the $X M M$-Newton Science Archive were analyzed to study the hot gas component.

Results. We estimate the cluster redshift $\langle z\rangle=0.177$, a high line-of-sight (LOS) velocity dispersion, $\sigma_{V} \sim 1350 \mathrm{~km} \mathrm{~s}^{-1}$, and the X-ray temperature $k T \sim 6.4 \mathrm{keV}$. Both our optical and X-ray analyses reveal complex dynamical activity. The analysis of the 2D galaxy distribution reveals the presence of two density peaks, one to the east and the other to the west (E and W peaks). Using the full 3D information we detect a high-velocity $\left(\Delta V_{\text {rf.LOS }} \sim 3000 \mathrm{~km} \mathrm{~s}^{-1}\right)$, low-mass $\left(\sigma_{V} \sim 200-500 \mathrm{~km} \mathrm{~s}^{-1}\right)$ group at the position of the 2D E peak. For the main system we compute a velocity dispersion $\sigma_{V} \sim 1000-1200 \mathrm{~km} \mathrm{~s}^{-1}$. In the assumption of a bimodal system we estimate a mass $M_{\text {sys }}=1.5-2.9 \times 10^{15} h_{70}^{-1} M_{\odot}$. The X-ray morphological analysis, which is based on power ratios, centroid shifts, and concentration parameter, confirms that Abell 2254 is a dynamically disturbed cluster. The X-ray isophotes are elongated in the east direction, in agreement with a merger in the post core-crossing phase. A simple bimodal model finds that data are consistent with a bound, outgoing subcluster observed a few fractions of Gyr after the core crossing. However, both optical and X-ray analyses suggest that the main system is, in turn, a nonrelaxed structure, indicating north-south as a possible direction for a past accretion.

Conclusions. We conclude that Abell 2254, for its mass and merging structure, fits well among the typical clusters with radio halos. We briefly discuss how the particular irregularity of the radio halo might be linked to the complexity of the Abell 2254 structure.
\end{abstract}

Key words. X-rays: galaxies: clusters - galaxies: kinematics and dynamics - galaxies: clusters: general galaxies: clusters: individual: Abell 2254

\section{Introduction}

Merging processes constitute an essential ingredient for the evolution of galaxy clusters (see Feretti et al. 2002 for a review). An interesting aspect of these phenomena is the possible connection between cluster mergers and extended, diffuse radio sources: halos and relics. The synchrotron radio emission of these sources demonstrates the existence of large-scale cluster magnetic fields and of widespread relativistic particles. Cluster mergers have been proposed as providing the large amount of energy necessary for electron re-acceleration to relativistic energies and for magnetic field amplification (Tribble 1993; Feretti 1999; Feretti 2002; Sarazin 2002). Radio relics ("radio gischts" as referred to by Kempner et al. 2004), which are polarized and elongated radio sources located in the cluster peripheral regions, seem to be directly associated with merger shocks (e.g., Ensslin et al. 1998; Roettiger et al. 1999; Ensslin \& Gopal-Krishna 2001; Hoeft et al. 2004). Radio halos are unpolarized sources that permeate the cluster volume in a similar way to the X-ray emitting gas of the intracluster medium (hereafter ICM). Radio halos are more likely to be associated with the turbulence following a cluster merger, although the precise radio formation scenario remains unclear (re-acceleration vs. hadronic models, e.g., Brunetti et al. 2009; Ensslin et al. 2011).

Recent semi-analytical calculations in the framework of the turbulent re-acceleration scenario have allowed the expectations for the statistical properties of giant radio halos to be derived, in agreement with present observation that halos are found in very massive clusters (Cassano \& Brunetti 2005; Cassano et al. 2006). Very recently, a unified halo-relic model has been presented in 
the framework of hadronic models where the time-dependence of the magnetic fields and of the cosmic ray distributions is taken into account to explain the observational properties of both halos and (most) relics (Keshet 2010). In this model the ICM magnetization is triggered by a merger event, in part but probably not exclusively in the wake of merger shocks.

Unfortunately, one has been able to study these phenomena only recently because there is now a sufficiently large data base, i.e. few dozen clusters hosting diffuse radio sources up to $z \sim 0.5$ (e.g., Giovannini et al. 1999; see also Giovannini \& Feretti 2002; Feretti 2005; Venturi et al. 2008; Bonafede et al. 2009; Giovannini et al. 2009). It is expected that new radio telescopes will strongly increase the number of diffuse sources (e.g., LOFAR, Cassano et al. 2010a) and allow the study of diffuse radio emission in low X-ray luminosity clusters so as to distinguish theories of halo formation (e.g., Cassano et al. 2005; Ensslin et al. 2011). The study of galaxy clusters with radio emission offers a unique tool for estimating the strength and structure of large-scale magnetic fields, which might have important cosmological implications (see Dolag et al. 2008, and Ferrari et al. 2008 for recent reviews). In particular, the study of clusters with radio halos/relics will likely contribute to quantifying the effect of the nonthermal pressure to the estimate of mass and temperature in galaxy clusters (e.g., Loeb \& Mao 1994; Markevitch 2010) and, more generally, the thermal and nonthermal effects of cluster mergers on global properties and cosmological parameters (e.g., Sarazin 2004).

From the observational point of view, there is growing evidence of a connection between diffuse radio emission and cluster mergers, since up to now diffuse radio sources have only been detected in merging systems (see Cassano et al. 2010b). In most cases the cluster's dynamical state has been derived from X-ray observations (Schuecker et al. 2001; Buote 2002; Cassano et al. $2010 b)$. Optical data are a powerful way to investigate the presence and the dynamics of cluster mergers, too (e.g., Girardi \& Biviano 2002). The spatial and kinematical analysis of member galaxies allow us to reveal and measure the amount of substructure, and to detect and analyze possible premerging clumps or merger remnants. This optical information is very complementary to X-ray information since galaxies and the ICM react on different timescales during a merger (see, e.g., the numerical simulations by Roettiger et al. 1997). In this context, we are conducting an intensive observational and data analysis program to study the internal dynamics of clusters with diffuse radio emission by using member galaxies (DARC - dynamical analysis of radio clusters - project, see Girardi et al. 2007 ${ }^{1}$ ).

During our observational program, we conducted an intensive study of the cluster Abell 2254 (hereafter A2254). A2254 is a very rich, $\mathrm{X}$-ray-luminous cluster: Abell richness class $=2$ (Abell et al. 1989); $L_{X}(0.1-2.4 \mathrm{keV})=7.19 \times 10^{44} h_{50}^{-2} \mathrm{erg} \mathrm{s}^{-1}$ (Ebeling et al. 1996). Optically, the cluster is classified as a Rood-Sastry morphological type "B", i.e. binary (Struble \& Rood 1987). The cluster redshift reported in the literature refers to $z=0.178$ of the brightest cluster galaxy (Crawford et al. 1995).

Evidence of an extended, diffuse radio source was reported by Owen et al. (1999) and of a radio halo with a projected size of $\sim 5^{\prime}$ is unambiguously shown by Giovannini et al. (1999). Both the radio and the X-ray emission (VLA $1.4 \mathrm{GHz}$ and ROSAT HRI data, respectively) show a very clumpy and irregular structure (Govoni et al. 2001b), with a radio power at $1.4 \mathrm{GHz}$ of

\footnotetext{
${ }^{1}$ See also the web site of the DARC project

http://adlibitum.oat.ts.astro.it/girardi/darc.
}

$P_{1.4 \mathrm{GHz}}=2.9 \times 10^{24} h_{70}^{-2} \mathrm{~W} \mathrm{~Hz}^{-1}$ and a large linear size of $\sim 0.9 h_{70}^{-1} \mathrm{Mpc}$.

We included this cluster in our DARC sample and obtained new spectroscopic and photometric data from the Telescopio Nazionale Galileo (TNG) and the Isaac Newton Telescope (INT), respectively. Our present analysis is based on these optical data, Subaru imaging data, and XMM-Newton Science Archive data, too.

This paper is organized as follows. We present optical data and the cluster catalog in Sect. 2. We present our results for the cluster structure based on optical and X-ray data in Sects. 3 and 4, respectively. We discuss these results and present our conclusions in Sect. 5.

Unless otherwise stated, we indicate errors at the $68 \%$ confidence level (hereafter c.l.). Throughout this paper, we use $H_{0}=70 \mathrm{~km} \mathrm{~s}^{-1} \mathrm{Mpc}^{-1}$ and $h_{70}=H_{0} /\left(70 \mathrm{~km} \mathrm{~s}^{-1} \mathrm{Mpc}^{-1}\right)$ in a flat cosmology with $\Omega_{0}=0.3$ and $\Omega_{\Lambda}=0.7$. In the adopted cosmology, $1^{\prime}$ corresponds to $\sim 180 h_{70}^{-1} \mathrm{kpc}$ at the cluster redshift.

\section{Galaxy data and catalog}

\subsection{Spectroscopic observations}

Multi-object spectroscopic observations of A2254 were carried out at the TNG telescope in May and October 2009 and in March 2010. We used DOLORES/MOS with the LR-B Grism 1, yielding a dispersion of $187 \AA / \mathrm{mm}$. We used the $2048 \times 2048$ pixels E2V CCD, with a pixel size of $13.5 \mu \mathrm{m}$. In total, we observed four MOS masks for a total of 142 slits. Total exposure times were $3600 \mathrm{~s}$ for two masks and $5400 \mathrm{~s}$ for the other two masks.

Wavelength calibration was performed using helium and neon-mercury lamps. Reduction of spectroscopic data was carried out with the IRAF $^{2}$ package. Radial velocities were determined using the cross-correlation technique (Tonry \& Davis 1979) implemented in the RVSAO package (developed at the Smithsonian Astrophysical Observatory Telescope Data Center). Each spectrum was correlated against five templates for a variety of galaxy spectral types: E, S0, Sa, Sb, and Sc (Kennicutt 1992). The template producing the highest value of $\mathcal{R}$, i.e., the parameter given by RVSAO and related to the signal-to-noise ratio of the correlation peak, was chosen. Moreover, all spectra and their best correlation functions were examined visually to verify the redshift determination. In three cases (IDs. 24, 35, and 60; see Table 1), we assumed the EMSAO redshift was a reliable estimate of the redshift. Our spectroscopic catalog lists 128 galaxies in the field of A2254.

The formal errors as given by the cross-correlation are known to be smaller than the true errors (e.g., Malumuth et al. 1992; Bardelli et al. 1994; Ellingson \& Yee 1994; Quintana et al. 2000). Duplicate observations for the same galaxy allowed us to estimate the true intrinsic errors in data of the same quality taken with the same instrument (e.g. Barrena et al. 2009). Here we have double determinations for seven galaxies, thus we decided to apply the procedure that had already been applied in the above study and found that true errors are larger than formal cross-correlation errors by a factor of 2.2. For the seven galaxies with two redshift estimates, we used the mean of the two measurements and the corresponding errors. As for the radial velocities estimated through EMSAO we assumed the largest between

2 IRAF is distributed by the National Optical Astronomy Observatories, which are operated by the Association of Universities for Research in Astronomy, Inc., under cooperative agreement with the National Science Foundation. 


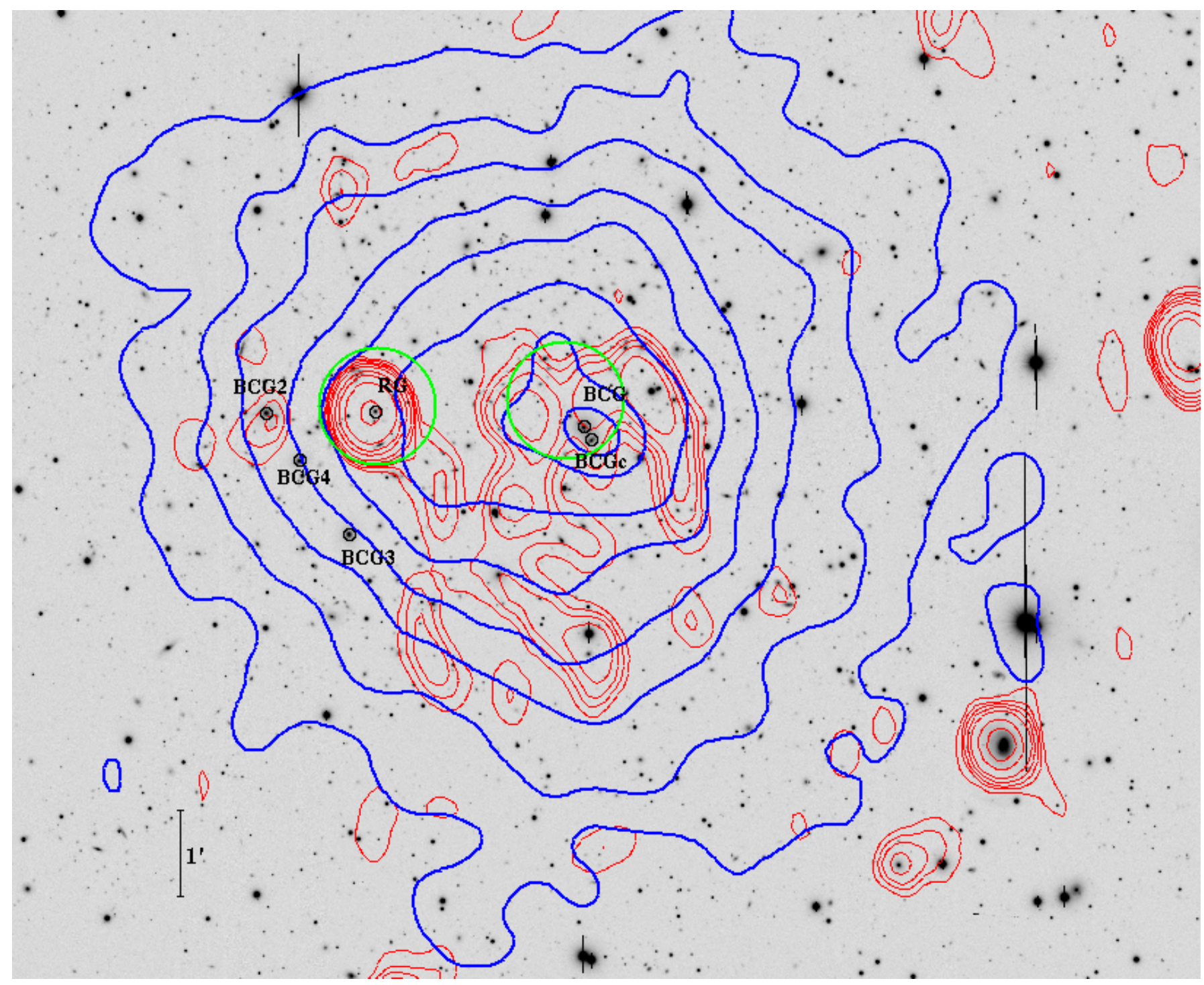

Fig. 1. Subaru $V$-band image of the cluster A2254 (north at the top and east to the left) with, superimposed, the contour levels of the XMM archival image (thick/blue contours; photons in the energy range $0.5-2 \mathrm{keV}$ ) and the contour levels of a VLA radio image at $1.4 \mathrm{GHz}$ (thin/red contours, see Govoni et al. 2001b). Labels indicate galaxies cited in the text. The two (green) circles indicate the positions of the $\mathrm{E}$ and $\mathrm{W}$ peaks in the galaxy distribution from the 2D-DEDICA analysis (Subaru sample, with $i^{\prime} \leq 20.5 \mathrm{mag}$, see Table 2).

the formal error and $100 \mathrm{~km} \mathrm{~s}^{-1}$, and the median value of the $c z$ errors for the whole catalog, taking the above correction into account, is $66 \mathrm{~km} \mathrm{~s}^{-1}$.

\subsection{INT observations}

Our photometric observations were carried out with the Wide Field Camera (WFC), mounted at the prime focus of the $2.5 \mathrm{~m}$ INT telescope. We observed A2254 in $g^{\prime}, r^{\prime}$, and $i^{\prime}$ SDSS filters in photometric conditions and a seeing of $\sim 1.6^{\prime \prime}$.

The WFC consists of a four-CCD mosaic covering a $33^{\prime} \times 33^{\prime}$ field of view, with only a $20 \%$ marginally vignetted area. We took twelve exposures of $600 \mathrm{~s}$ in $g^{\prime}$ filter, nine exposures of $450 \mathrm{~s}$ in $r^{\prime}$ filter, and nine exposures of $400 \mathrm{~s}$ in $i^{\prime}$ filter, so a total of $7200 \mathrm{~s}$ in $g^{\prime}$ filter, $4050 \mathrm{~s}$ in $r^{\prime}$ and $3600 \mathrm{~s}$ in $i^{\prime}$ band. The observations were performed making a dithering pattern. This observing procedure allowed us to build a "supersky" frame that was used to correct our images for fringing patterns (Gullixson 1992). In addition, the dithering helped us clean cosmic rays and avoid the effects of gaps between the CCDs in the final full mosaic images. Another problem associated with the wide field frames is the distortion of the field. To match the photometry of several filters, a good astrometric solution is needed to take these distortions into account. Using the imcoords IRAF tasks and taking as a reference the USNO B1.0 catalog, we were able to obtain an accurate astrometric solution (rms $\sim 0.3^{\prime \prime}$ ) across the full frame. Only on the northeast edge of the image, at $\sim 17^{\prime}$ from the cluster center, were we unable to a good enough astrometric solution. To guarantee a good match between the $g^{\prime}, r^{\prime}$, and $i^{\prime}$ catalogs, this marginal region was removed from the master catalog. The photometric calibration was performed by observing the SA108 standard Landolt field (Landolt 1992), which is also calibrated in the SDSS photometric system (Smith 2002).

We finally identified galaxies in our $g^{\prime}, r^{\prime}$ and $i^{\prime}$ images and measured their magnitudes with the SExtractor package (Bertin \& Arnouts 1996) and AUTOMAG procedure. Objects were identified by requiring that they cover a certain minimum 
area and have a number of counts above a limiting threshold taking the sky local background as a reference. The limiting size and flux were 16 pixels and 1.5 standard deviation above the sky counts level, respectively. The selected limiting size corresponds to an apparent size of $1.3^{\prime \prime}$, which is about the minimum seeing size during the observations. We also performed visual inspections of the frames in order to deal with the best combination of the above parameters that remove spurious objects from the catalogs.

In a few cases (e.g., close pairs of galaxies, galaxies very close to bright stars, or those close to defects of the CCD), the standard SExtractor photometric procedure failed, at which point we computed magnitudes by hand. This method consisted of measuring fluxes assuming a galaxy profile of a typical elliptical galaxy and scaling it to the maximum observed value. The integration of this profile provided an estimate of the magnitude. This method is similar to PSF photometry, but assumes an earlytype galaxy profile, which is more appropriate in this case.

As a final step, we estimated and corrected the Galactic extinction $A_{g^{\prime}} \sim 0.21, A_{r^{\prime}} \sim 0.15$ and $A_{i^{\prime}} \sim 0.10$ using Schlegel et al. (1998) reddening and extinction maps computed from IRAS and COBE/DIRBE data. We estimated that our photometric sample is complete down to $g^{\prime}=23.4(24.3), r^{\prime}=22.1$ (23.3), and $i^{\prime}=21.9$ (23.3) for $S / N=5$ (3) within the observed field. We assigned magnitudes to all galaxies of our spectroscopic catalog.

\subsection{Subaru images}

Subaru Suprime-Cam data were retrieved from the SMOKA archive $^{3}$. Following the same steps as adopted by Nonino et al. (2009), the images in $V$-band and $i^{\prime}$-band were coadded and reduced to cover a $30^{\prime} \times 30^{\prime}$ field of view. Seeing conditions were $\sim 0.8^{\prime \prime}-1.0^{\prime \prime}$, with a total exposure time of $1200 \mathrm{~s}$ in $V$-band, and $1920 \mathrm{~s}$ in $i^{\prime}$-band. The astrometric solutions were obtained using 2MASS as reference.

Unfortunately, the observations in both filters were collected under nonphotometric conditions, as shown from the relative photometric analysis. Therefore, the final coadded images were not calibrated with standard stars. For the use we make in this study, i.e. the $2 \mathrm{D}$ analysis of substructure, noncalibrated magnitudes are sufficient, and we refer to them as $i_{\text {obs }}^{\prime}$ and $V_{\text {obs }}$ (see Sect. 3.4 for other details).

\subsection{Galaxy catalog}

Table 1 lists the velocity catalog (see also Fig. 2): identification number of each galaxy and member galaxies, ID and IDm (Cols. 1 and 2, respectively); right ascension and declination, $\alpha$ and $\delta$ (J2000, Col. 3); $g^{\prime}, r^{\prime}$, and $i^{\prime}$ INT magnitudes (Cols. 4-6); heliocentric radial velocities, $v=c z_{\odot}($ Col. 7) with errors, $\Delta v$ (Col. 8).

\subsection{Individual galaxies}

No evident dominant galaxy is present in the cluster since the brightest galaxy in our catalog (ID. $53, r^{\prime}=16.22$, hereafter $\mathrm{BCG}$ ) is less than 0.5 mag brighter than the following brightest galaxies (IDs. 117, 102, 112 with $r^{\prime}=16.64,16.66$, and 16.67, respectively). These bright galaxies all lie in the east region of the cluster; we refer to them as BCG2, BCG3, and BCG4.
Table 1. Velocity catalog of 128 spectroscopically measured galaxies in the field of the cluster A2254.

\begin{tabular}{|c|c|c|c|c|c|c|c|}
\hline ID & IDm & $\alpha, \delta(\mathrm{J} 2000)$ & $g^{\prime}$ & $r^{\prime}$ & $i^{\prime}$ & $\begin{array}{c}v \\
(\mathrm{~km}\end{array}$ & $\begin{array}{l}\Delta v \\
\left.\mathrm{~s}^{-1}\right)\end{array}$ \\
\hline 1 & 1 & $171724.62,+194159.6$ & 18.52 & 17.45 & 16.98 & 51510 & 53 \\
\hline 2 & 2 & $171725.92,+193954.7$ & 20.72 & 19.63 & 19.25 & 52688 & 68 \\
\hline 3 & - & $171725.97,+194325.3$ & 18.62 & 17.27 & 16.84 & 72033 & 64 \\
\hline 4 & - & $171726.26,+194220.5$ & 18.90 & 17.70 & 17.41 & 70536 & 66 \\
\hline 5 & 3 & $171726.59,+194131.6$ & 18.89 & 18.17 & 17.96 & 52741 & 77 \\
\hline 6 & 4 & $171727.62,+194214.8$ & 19.31 & 18.15 & 17.74 & 52931 & 46 \\
\hline 7 & - & $171728.94,+194018.1$ & 21.59 & 20.08 & 19.37 & 165420 & 106 \\
\hline 8 & 5 & $171730.10,+194330.0$ & 19.88 & 18.71 & 18.30 & 53232 & 70 \\
\hline 9 & 6 & $171731.68,+194206.1$ & 19.28 & 18.16 & 17.76 & 53803 & 55 \\
\hline 10 & - & $171732.35,+194313.4$ & 20.82 & 19.89 & 19.46 & 78076 & 84 \\
\hline 11 & 7 & $171733.53,+194107.1$ & 19.68 & 19.11 & 18.99 & 53136 & 90 \\
\hline 12 & - & $171733.55,+194242.1$ & 20.26 & 19.21 & 18.94 & 71797 & 64 \\
\hline 13 & - & $171734.37,+194248.6$ & 17.50 & 16.51 & 16.12 & 36622 & 37 \\
\hline 14 & 8 & $171735.35,+194242.8$ & 19.70 & 18.87 & 18.56 & 54559 & 103 \\
\hline 15 & 9 & $171735.95,+193927.4$ & 20.10 & 19.10 & 18.81 & 52006 & 139 \\
\hline 16 & 10 & $171736.43,+194107.8$ & 19.71 & 18.67 & 18.32 & 513 & 42 \\
\hline 17 & 11 & $171736.46,+193902.2$ & 19.32 & 18.57 & 18.26 & 546 & 73 \\
\hline 18 & 12 & 171736.7 & 20.80 & 19.65 & 19.28 & 54105 & 70 \\
\hline 19 & 13 & $171737.03,+194111.4$ & 19.44 & 18.75 & 18.35 & 77 & 53 \\
\hline 20 & - & $171737.87,+19$ & 21.04 & 19.72 & 19.36 & 62831 & 62 \\
\hline 21 & - & $171738.04,+194004.4$ & 20.20 & 18.95 & 18.72 & 70 & 46 \\
\hline 22 & 14 & $171738.04,+194455.7$ & 20.37 & 19.27 & 18.89 & 551 & 101 \\
\hline 23 & 15 & $171738.21,+194003.7$ & 21.76 & 20.84 & 19.88 & 52576 & 150 \\
\hline 24 & 16 & $171738.26,+193846.3$ & 21.17 & 20.03 & 19.47 & 53964 & 100 \\
\hline 25 & 17 & $171738.95,+194001.6$ & 19.37 & 18.20 & 17.79 & 52664 & 62 \\
\hline 26 & - & $171739.10,+194133.0$ & 21.20 & 19.77 & 19.21 & 149288 & 48 \\
\hline 27 & 18 & $171739.65,+194106.7$ & 20.22 & 19.05 & 18.79 & 51500 & 121 \\
\hline 28 & 19 & $171739.70,+193802.8$ & 20.52 & 19.45 & 19.07 & 540 & 6 \\
\hline 29 & 20 & $171739.84,+193810.7$ & 20.67 & 19.64 & 19.30 & 526 & \\
\hline 30 & 21 & $171739.84,+193810.7$ & 20.67 & 19.64 & 19.30 & 54818 & 90 \\
\hline 31 & 22 & $171740.03,+194052.0$ & 19.43 & 18.35 & 17.98 & $52 \varepsilon$ & 33 \\
\hline 32 & 23 & 17174 & 20.52 & 19.86 & 19.63 & & 240 \\
\hline 33 & 24 &,+1 & 20.33 & 19.16 & 18.70 & 54 & 66 \\
\hline 34 & 25 & $171740.68,+193952.2$ & 20.98 & 19.84 & 19.39 & 54 & 103 \\
\hline 35 & 26 & $171741.02,+194353.8$ & 20.16 & 19.37 & 19.06 & 515 & 100 \\
\hline 36 & 27 & $171741.33,+193952.2$ & 19.78 & 19.06 & 18.81 & 53081 & 128 \\
\hline 37 & - & $171741.38,+194142.0$ & 18.73 & 18.04 & 17.75 & 62608 & 55 \\
\hline 38 & 28 & $171741.57,+194107.4$ & 19.01 & 18.04 & 17.81 & 54845 & 66 \\
\hline 39 & 29 & $171742.34,+194239.2$ & 17.98 & 17.18 & 16.90 & 54521 & 77 \\
\hline 40 & 30 & $171743.01,+194226.3$ & 19.19 & 18.05 & 17.58 & 53870 & 62 \\
\hline 41 & 31 & $171743.06,+194144.2$ & 18.91 & 17.98 & 17.57 & 52292 & 35 \\
\hline 42 & 32 & $171743.06,+194351.6$ & 18.75 & 17.69 & 17.41 & 53468 & 41 \\
\hline 43 & 33 & $171743.20,+194459.6$ & 19.16 & 18.28 & 17.99 & 50532 & 51 \\
\hline 44 & 34 & $171743.61,+193920.5$ & 19.25 & 18.10 & 17.77 & 52880 & 66 \\
\hline 45 & 35 & $171743.61,+194242.1$ & 19.58 & 18.47 & 18.09 & 535 & 44 \\
\hline 46 & 36 & 17 & 21.34 & 20.46 & 20.07 & & 99 \\
\hline 47 & 37 & , 193924.8 & 20.90 & 19.97 & 19.65 & 52533 & 189 \\
\hline 48 & 38 & $171743.80,+194112.1$ & 19.26 & 18.24 & 17.96 & 55744 & 62 \\
\hline 49 & 39 & $171743.87,+194143.4$ & 19.42 & 18.10 & 17.93 & 52024 & 66 \\
\hline 50 & 40 & $171745.53,+194040.1$ & 17.90 & 17.01 & 16.74 & 54. & 53 \\
\hline 51 & 41 & $171745.62,+194157.1$ & 21.28 & 20.09 & 19.72 & 53513 & 110 \\
\hline 52 & 42 & $171745.65,+193813.6$ & 19.31 & 18.33 & 17.93 & 53918 & 66 \\
\hline 53 & 43 & $171745.86,+194048.4$ & 17.44 & 16.22 & 15.73 & 52963 & 41 \\
\hline 54 & 44 & $171745.86,+194147.4$ & 19.69 & 18.62 & 18.23 & 51347 & 55 \\
\hline 55 & 45 & $171745.86,+194045.8$ & 20.66 & 19.76 & 19.21 & 53276 & 75 \\
\hline 56 & 46 & $171745.91,+193941.4$ & 19.45 & 18.29 & 17.89 & 53264 & 44 \\
\hline 57 & 47 & $171746.30,+194220.9$ & 20.35 & 19.24 & 18.84 & 52108 & 130 \\
\hline 58 & 48 & $171746.51,+194242.5$ & 20.45 & 19.49 & 19.01 & 53167 & 59 \\
\hline 59 & 49 & $171746.58,+194005.9$ & 19.90 & 19.27 & 18.40 & 53069 & 55 \\
\hline 60 & - & 171 & 19.43 & 18.83 & 18.58 & 23305 & 170 \\
\hline 61 & 50 & $171746.97,+193931.3$ & 18.94 & 17.81 & 17.36 & 50067 & 66 \\
\hline 62 & - & $171747.59,+194129.4$ & 20.50 & 19.78 & 19.51 & 89174 & 158 \\
\hline 63 & 51 & $171747.69,+194415.4$ & 19.27 & 18.46 & 18.20 & 55316 & 66 \\
\hline
\end{tabular}

\footnotetext{
${ }^{3}$ http://smoka.nao.ac.jp/SUPsearch
} 
Table 1. continued.

\begin{tabular}{|c|c|c|c|c|c|c|c|}
\hline ID & $\mathrm{IDm}$ & $\alpha, \delta(\mathrm{J} 2000)$ & $g^{\prime}$ & $r^{\prime}$ & $i^{\prime}$ & $\begin{array}{c}v \\
(\mathrm{~km}\end{array}$ & $\begin{array}{l}\Delta v \\
\left.\mathrm{~s}^{-1}\right)\end{array}$ \\
\hline 64 & 52 & $171747.78,+194135.9$ & 19.13 & 18.14 & 17.80 & 54202 & 59 \\
\hline 65 & 53 & $171747.95,+193700.8$ & 20.57 & 19.49 & 19.07 & 5387 & 59 \\
\hline 66 & 54 & $171748.02,+193851.4$ & 20.31 & 19.28 & 18.92 & 55179 & 46 \\
\hline 67 & 55 & $171749.78++194110.7$ & 19.82 & & 18.55 & 54240 & 77 \\
\hline 68 & 56 & $171749.82,+194118.2$ & 21.19 & 20.17 & 19.85 & 51305 & \\
\hline 69 & 57 & $171750.02,+193907.6$ & 18.15 & 16.98 & 16.57 & 54695 & 39 \\
\hline 70 & 58 & $171750.81,+194143.4$ & 18.86 & 17.76 & 17.35 & 51441 & 37 \\
\hline 71 & 59 & $171750.95,+194112.5$ & 20.47 & 19.41 & 19.28 & 53289 & 55 \\
\hline 72 & 60 & $171751.22,+194238.5$ & 18.84 & 18.06 & 17.84 & 49237 & 79 \\
\hline 73 & 61 & $171751.48,+194036.1$ & 19.47 & 18.31 & 17.95 & 52695 & 79 \\
\hline 74 & - & $171751.58,+194252.9$ & 18.61 & 17.37 & 16.94 & 62934 & 70 \\
\hline 75 & 62 & $171751.65,+194120.4$ & 19.78 & 18.57 & 18.70 & 51961 & 86 \\
\hline 76 & 63 & $171752.13,+194019.6$ & 19.39 & 18.26 & 17.85 & 52167 & 59 \\
\hline 77 & 64 & $171752.61,+194008.0$ & 20.18 & 19.04 & 18.76 & 52308 & 46 \\
\hline 78 & 65 & $171753.04,+194424.0$ & 19.94 & 18.82 & 18.45 & 51433 & 66 \\
\hline 79 & 66 & $171753.21,+193847.8$ & 18.73 & 17.68 & 17.34 & 50868 & 46 \\
\hline 80 & 67 & $171753.52,+194025.0$ & 20.20 & 19.10 & 18.68 & 56052 & 86 \\
\hline 81 & 68 & $12,194147.8$ & 19.13 & 18.00 & 17.64 & 52069 & 48 \\
\hline 82 & 69 & $171754.17,+1$ & 21.78 & 20.77 & 20.30 & 53213 & 108 \\
\hline 83 & 70 & $171754.84,+193814.3$ & 19.58 & 18.70 & 18.37 & 53855 & 64 \\
\hline 84 & 71 & $171754.91,+193904.3$ & 20.28 & 19.16 & 18.74 & 53449 & 75 \\
\hline 85 & 72 & $171754.96,+193929.2$ & 20.38 & 19.87 & 19.61 & 54529 & 174 \\
\hline 86 & 73 & $171755.06,+194016.0$ & 18.56 & 17.49 & 17.11 & 49834 & 62 \\
\hline 87 & 74 & $171755.06,+194210.8$ & 20.40 & 19.22 & 18.82 & 56376 & 66 \\
\hline 88 & 75 & $171755.08,+194110.7$ & 19.59 & 18.40 & 17.99 & 56400 & 48 \\
\hline 89 & 76 & $171755.46,+193752.0$ & 21.36 & 20.34 & 19.98 & 53505 & 101 \\
\hline 90 & 77 & $171755.75,+194153.2$ & 20.84 & 19.94 & 19.64 & 56368 & 97 \\
\hline 91 & 78 & $171755.78,+193941.4$ & 19.97 & 18.83 & 18.44 & 56515 & 59 \\
\hline 92 & 79 & $171755.78,+19$ & 19.18 & 18.60 & 18.37 & 52917 & 68 \\
\hline 93 & 80 & $171756.02,+194059.5$ & 18.93 & 17.81 & 17.41 & 53106 & 53 \\
\hline 94 & 81 &,+15 & 20.78 & 19.93 & 19.98 & 13 & 75 \\
\hline 95 & 82 & 17 & 19.64 & 18.49 & 18.05 & T & 37 \\
\hline 96 & 83 & 4143.4 & 20.13 & 19.15 & 18.82 & 55943 & 117 \\
\hline 97 & 84 & $171756.50,+193759.9$ & 20.34 & 19.25 & 18.86 & 53815 & 57 \\
\hline 98 & 85 & $171756.62,+194256.5$ & 19.35 & 18.18 & 17.82 & 54648 & 35 \\
\hline 99 & 86 & $171756.95,+194121.5$ & 19.97 & 18.92 & 18.59 & 54154 & 44 \\
\hline 100 & 87 & $171757.07,+194128.7$ & 18.67 & 17.54 & 17.16 & 53106 & 42 \\
\hline 101 & 88 & $171757.19,+193723.5$ & 20.86 & 19.86 & 19.61 & 52885 & 97 \\
\hline 102 & 89 & $171757.26,+193935.3$ & 17.79 & 16.66 & 16.25 & 51409 & 55 \\
\hline 103 & 90 & $171757.29,+194152.4$ & 18.92 & 17.78 & 17.38 & 52138 & 42 \\
\hline 104 & 91 & $171757.53,+194352.0$ & 18.91 & 17.75 & 17.59 & 53675 & 51 \\
\hline 105 & 92 & $171757.67,+194342.2$ & 19.98 & 19.06 & 19.05 & 53747 & 79 \\
\hline 106 & 93 & $171757.84,+194328.9$ & 19.01 & 18.45 & 18.17 & 54201 & 68 \\
\hline 107 & 94 & $171758.13,+193839.1$ & 18.47 & 17.59 & 17.41 & 50570 & 55 \\
\hline 108 & 95 & 171758 & 20.65 & 19.54 & 19.23 & 51708 & 62 \\
\hline 109 & - & 17 & 20 & 19.60 & 19 & 562 & 147 \\
\hline 10 & 96 & $1,+193723.2$ & 19.97 & 18.93 & 18.59 & 51871 & 70 \\
\hline 111 & 97 & 17175 & 19.34 & 18.19 & 17.73 & 56757 & 55 \\
\hline 112 & 98 & , +194025.7 & 17.77 & 16.67 & 16.26 & 53416 & 64 \\
\hline 113 & 99 & $171759.83,+194209.0$ & 19.57 & 18.92 & 18.70 & 51016 & 57 \\
\hline 114 & 100 & $171759.98,+194054.1$ & 20.66 & 19.89 & 19.57 & 53090 & 68 \\
\hline 115 & 101 & $171800.58,+194344.8$ & 20.94 & 20.04 & 19.64 & 52104 & 90 \\
\hline 116 & 102 & $171800.67,+194000.1$ & 20.92 & 19.80 & 19.40 & 54904 & 77 \\
\hline 117 & 103 & $171801.30,+194058.4$ & 17.74 & 16.64 & 16.21 & 54229 & 57 \\
\hline 118 & 104 & $171801.70,+193928.8$ & 19.27 & 18.13 & 17.72 & 52903 & 68 \\
\hline 119 & 105 & $171801.97,+194022.4$ & 19.56 & 18.53 & 18.08 & 51933 & 92 \\
\hline 120 & - & $171801.99,+193756.6$ & 20.09 & 19.31 & 18.97 & 70025 & 99 \\
\hline 121 & 106 & $171803.31,+194209.4$ & 19.80 & 18.70 & 18.34 & 55067 & 46 \\
\hline 122 & 107 & $171803.46,+193614.0$ & 20.19 & 19.12 & 18.73 & 51813 & 64 \\
\hline 123 & - & $171803.62,+193733.6$ & 21.07 & 19.97 & 19.51 & 122887 & 200 \\
\hline 124 & 108 & 21.4 & 19.72 & 19.58 & 19.50 & 50836 & 110 \\
\hline 125 & - & $171804.61,+193922.3$ & 19.71 & 18.28 & 17.80 & 105488 & 55 \\
\hline 126 & 109 & $171806.00,+193955.1$ & 19.51 & 18.52 & 18.17 & 52452 & 125 \\
\hline 127 & 110 & $171806.05,+193817.5$ & 18.38 & 17.29 & 16.90 & 51558 & 62 \\
\hline 128 & - & $171807.51,+193740.8$ & 19.72 & 18.71 & 18.36 & 96285 & 75 \\
\hline
\end{tabular}

The BCG is close to the peak of the X-ray emission and has a (projected) bright companion $\left(r^{\prime}=17.01\right.$, ID. 50 hereafter $\mathrm{BCGc}$ ). BCGc is characterized by [OII], $\mathrm{H} \beta$, and [OIII] emission spectral lines, strong $24 \mu$ MIPS-Spitzer emission ${ }^{4}$, UV emission $^{5}$, and faint radio emission (ID. 2 in Table 3 of Rizza et al. 2003). The Subaru images show that it is a spiral galaxy with a large bulge and an arm directed toward the BCG (see Fig. 3). We find quite a large relative velocity difference with respect to the BCG $\left(\sim 1400 \mathrm{~km} \mathrm{~s}^{-1}\right)$.

Figure 2 of Govoni et al. (2001b) suggests there are a few discrete radio sources. The brightest one - with a flux of $\sim 38 \mathrm{mJy}$ at $1.4 \mathrm{GHz}$ - lies in the northeast cluster region where the field is very crowded. The likely optical counterpart might be the cluster galaxy ID. 93 (hereafter "RG"), which has an optical spectrum typical of ellipticals. ID. 93, with an absolute magnitude $M_{\mathrm{R}} \sim-21.9$ and a radio power $\log P_{1.4 \mathrm{GHz}}\left(\mathrm{W} \mathrm{Hz}^{-1}\right) \sim 24.5$, can be accommodated among the radio galaxies in clusters (e.g., Ledlow \& Owen 1996; Bardelli et al. 2010). However, a very faint, extended object close to RG is revealed by the Subaru image (Fig. 4). Due to the present resolution in radio data, there is some doubt about the identification of RG as (uniquely) responsible for the whole radio emission of the bright discrete radio source. Figure 4 also shows the position of a point-like X-ray source and of an MIPS-Spitzer source.

\section{Analysis of the optical data}

\subsection{Member selection}

To select cluster members among the 128 galaxies with redshifts, we performed the 1D adaptive-kernel method (hereafter DEDICA, Pisani 1993, and 1996; see also Fadda et al. 1996; Girardi et al. 1996). We search for significant peaks in the velocity distribution at $>99 \%$ c.l. This procedure detects A2254 as a peak at $z \sim 0.177$ populated by 110 galaxies considered as fiducial cluster members (in the range $49237 \leq v \leq 56757 \mathrm{~km} \mathrm{~s}^{-1}$, see Fig. 5). As for the center of A2254, we adopted the position of the BCG $\left[\mathrm{RA}=17^{\mathrm{h}} 17^{\mathrm{m}} 45^{\mathrm{s}} .86\right.$, Dec $=+19^{\circ} 40^{\prime} 48.4^{\prime \prime}$ (J2000.0)]. The 18 nonmembers are 2 foreground and 16 background galaxies.

In the previous analyses of DARC clusters, we usually applied a second step in the member selection, which uses the combination of position and velocity information, i.e., the "shifting gapper" method by Fadda et al. (1996). This procedure rejects galaxies that are too far in velocity from the main body of galaxies within a fixed bin that shifts along the distance from the cluster center (adopted values are $1000 \mathrm{~km} \mathrm{~s}^{-1}$ in the cluster rest frame for the velocity cut and $0.6 h_{70}^{-1} \mathrm{Mpc}$, or large enough to include 15 galaxies, for the spatial shifting bin). The procedure is iterated until the number of cluster members converges to a stable value (see Fadda et al. for other details and their Fig. 1 for an example). Here, the formal application of the "shifting gapper" procedure would reject another four galaxies just at the border of the distribution of galaxies in the plane of the rest-frame velocity vs. projected clustercentric distance (see Fig. 6 - top panel). These galaxies are not spatially clustered and have the typical early-type galaxy colors. Therefore, we decided to not reject them from the analysis. Global cluster quantities are independent of this decision within the 1-sigma error. The "shifting gapper" procedure is completely empirical and has the advantage of being independent of hypotheses about the poorly

\footnotetext{
4 See at

http://sha.ipac. caltech.edu/applications/Spitzer/SHA/ ${ }^{5}$ http://galex.stsci.edu/GalexView/
} 


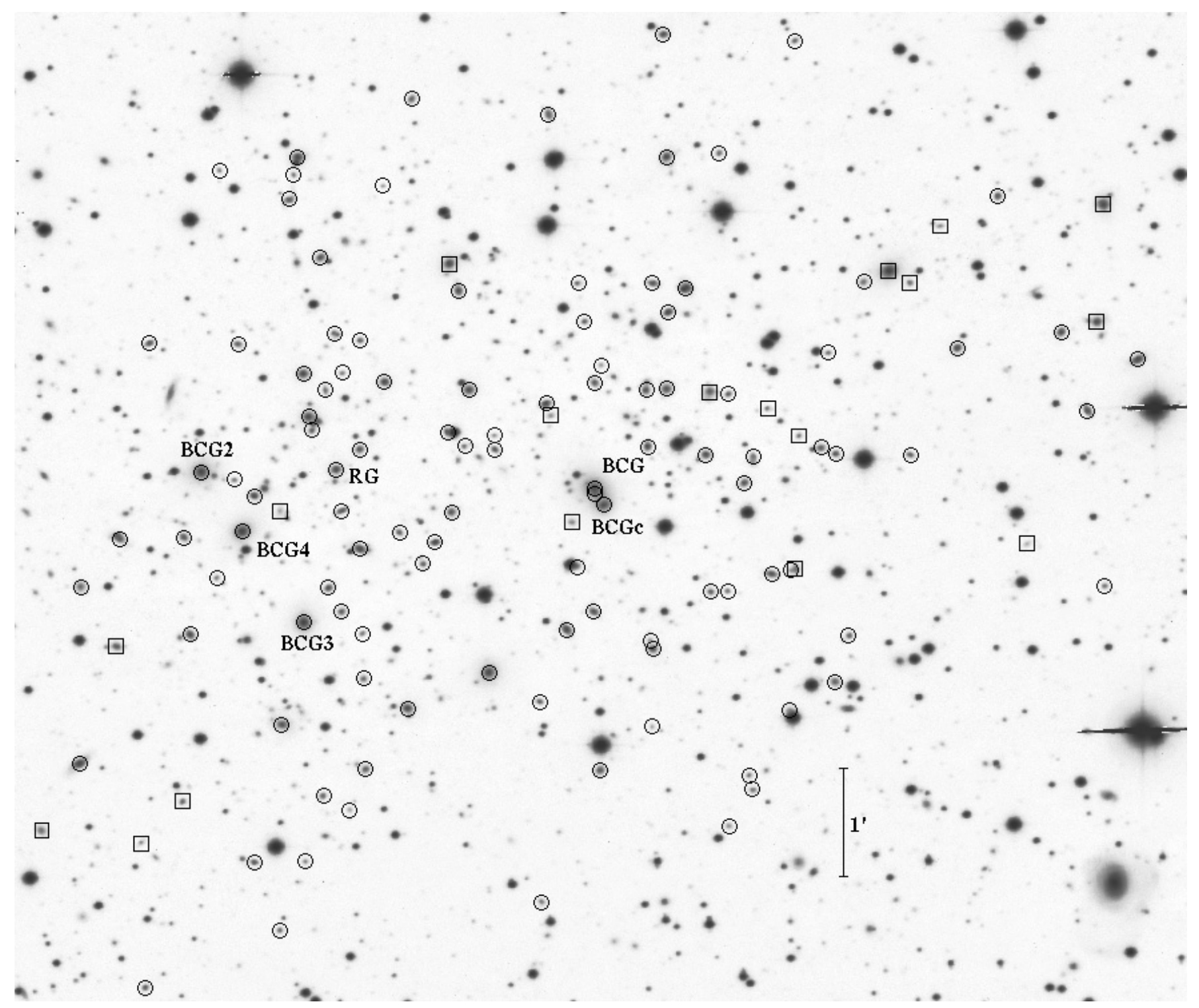

Fig. 2. INT $r^{\prime}$-band image of the cluster A2254 (north at the top and east to the left). Circles and squares indicate cluster members and nonmembers, respectively (see Table 1). Labels indicate cluster galaxies cited in the text.

known dynamical status of the cluster. Other available procedures combining position and velocity information are generally based on physical assumptions about the dynamical status of the cluster and the consequent possibility of appling the virial mass and/or the knowledge of the mass profile (e.g., den Hartog \& Katgert 1996; see Biviano et al. 2006, for a recent application). Therefore, applying these procedures is not very justified in the case of A2254, showing evidence of substructure (see in the following). Making an attempt in this direction, we use the value of the mass presented in Sect. 5.3, $M_{\text {sys }}$, and adopt a single cluster model as described by the NFW mass profile (Navarro et al. 1997; see Sect. 5.3 too) to construct the upper limits to the LOS velocities at projected distance $R$ from the cluster center (criterion (i) by den Hartog \& Katgert 1996). Figure 6 shows that the region of allowed velocities is roughly coincident with that occupied by our selected member galaxies. Thus, although in the case of the substructured cluster A2254 we do not expect to determine the detailed cluster membership, it supports the consistency between our adopted galaxy membership and the value we obtain for the mass estimate.

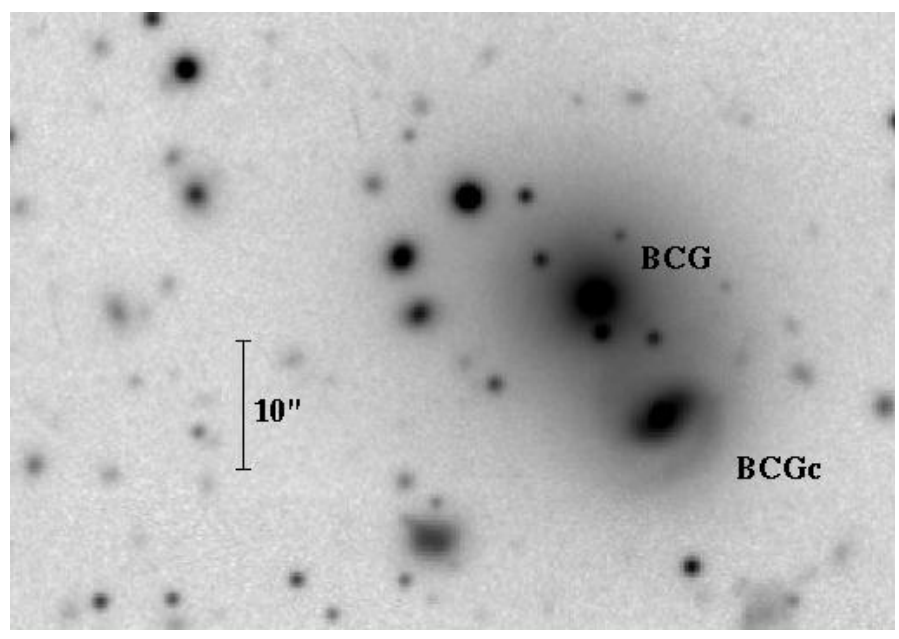

Fig. 3. Subaru $i^{\prime}$-band image of the very central cluster region. It highlights the BCG and its bright spiral companion BCGc (north at the top and east to the left). 


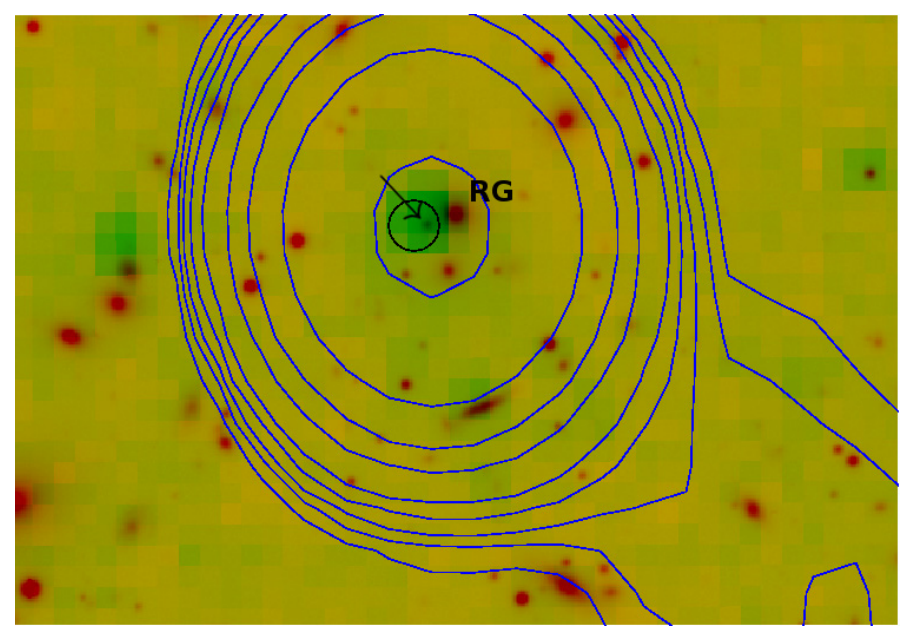

Fig. 4. Multiwavelength picture of the region around RG galaxy. The MIPS-Spitzer image (green color) is superimposed to the Subaru $i^{\prime}$ band image. The arrow indicates the faint extended object close to RG. The black circle indicate the position of the XMM point source. Contour levels of the radio image are shown in blue (Govoni et al. 2001b). North at the top and east to the left.

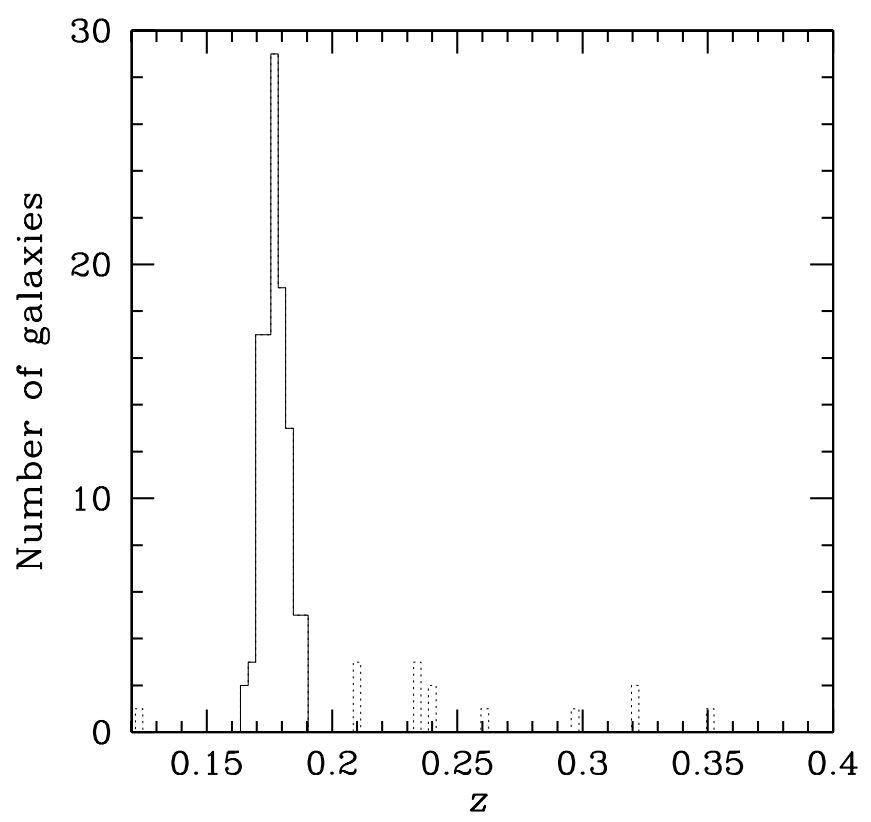

Fig. 5. Redshift galaxy distribution. The solid line histogram refers to the 110 galaxies assigned to A2254 according to the DEDICA reconstruction method.

\subsection{Global cluster properties}

By applying the biweight estimator to the 110 cluster members (Beers et al. 1990, ROSTAT software), we compute a mean cluster redshift of $\langle z\rangle=0.1772 \pm 0.0004$, i.e. $\langle v\rangle=(53128 \pm$ $125) \mathrm{km} \mathrm{s}^{-1}$. We estimate the LOS velocity dispersion, $\sigma_{V}$, by using the biweight estimator and applying the cosmological correction and the standard correction for velocity errors (Danese et al. 1980). We obtain $\sigma_{V}=1340_{-84}^{+101} \mathrm{~km} \mathrm{~s}^{-1}$, where errors are estimated through a bootstrap technique.

To evaluate the robustness of the $\sigma_{V}$ estimate, we analyze the velocity dispersion profile (Fig. 6). The integral profile shows an

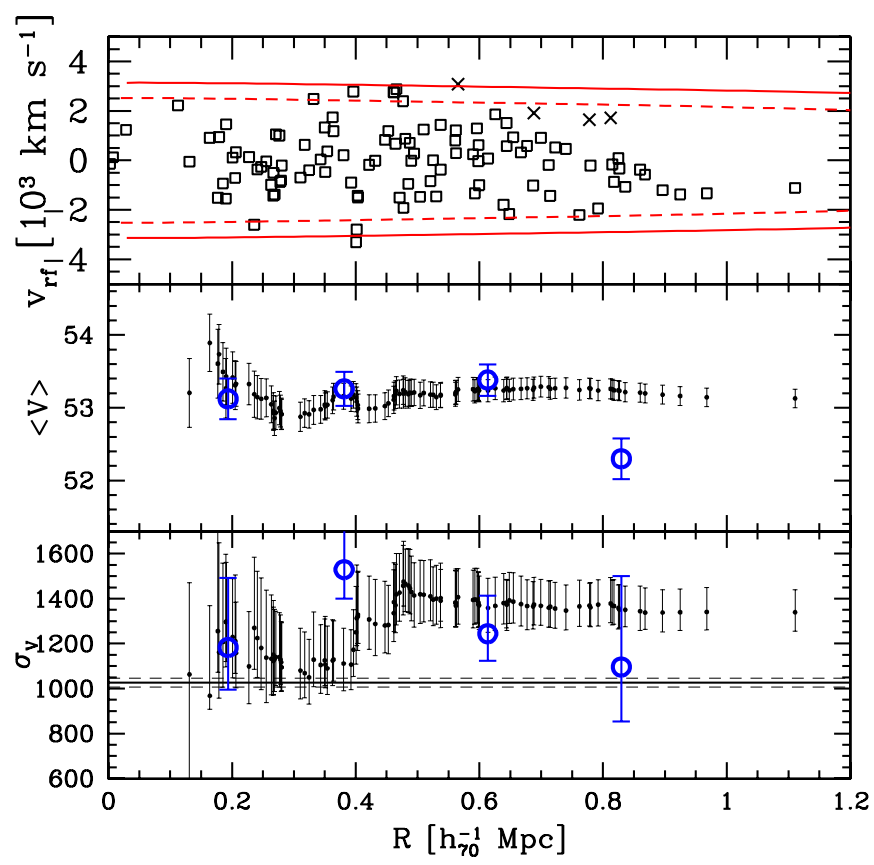

Fig. 6. Top panel: rest-frame velocity vs. projected clustercentric distance for the 110 member galaxies of the fiducial sample (Fig. 5). Crosses indicate galaxies formally rejected as interlopers by the "shifting gapper" procedure, but here considered in a more conservative view. The cluster center coincides with the position of the BCG. Red solid (dashed) curves enclose the region of allowed values for member galaxies according to the criterion of den Hartog \& Katgert (1996) and using the upper (lower) limit of our mass estimate computed in the following (see Sect. 5.3). Middle and bottom panels: differential (big circles) and integral (small points) profiles of mean velocity and LOS velocity dispersion, respectively. For the differential profiles, we plot the values for four annuli from the center of the cluster, each $0.25 h_{70}^{-1} \mathrm{Mpc}$ (large blue symbols). For the integral profiles, the mean and dispersion at a given (projected) radius from the cluster-center is estimated by considering all galaxies within that radius - the first value computed on the five galaxies closest to the center. The error bands at the $68 \%$ c.l. are also shown. In the bottom panel, the horizontal line represents the X-ray temperature with the respective errors transformed in $\sigma_{V}$ assuming the density-energy equipartition between ICM and galaxies, i.e. $\beta_{\text {spec }}=1$ (see Sect. 5).

enhancement at $\sim 0.4-0.5 h_{70}^{-1} \mathrm{Mpc}$ from the cluster center and then flattens. This suggests that a robust value of $\sigma_{V}$ is asymptotically reached in the external cluster regions, as found for the majority of nearby clusters (e.g., Fadda et al. 1996; Girardi et al. 1996). As for the enhancement at $\sim 0.4-0.5 h_{70}^{-1} \mathrm{Mpc}$, it is likely due to contamination of the galaxies of a secondary clump (see Sects. 3.4 and 3.5); see also, e.g., Girardi et al. (1996) and Barrena et al. (2007) for examples in other clusters.

\subsection{Velocity distribution}

We analyzed the velocity distribution to search for possible deviations from Gaussianity that might provide important signatures of complex dynamics. For the following tests, the null hypothesis is that the velocity distribution is a single Gaussian.

We estimate three shape estimators, i.e., the kurtosis, the skewness, and the scaled tail index (see, e.g., Bird \& Beers 1993). There is no evidence of departures from the Gaussianity (see Table 2 of Bird \& Beers 1993). 


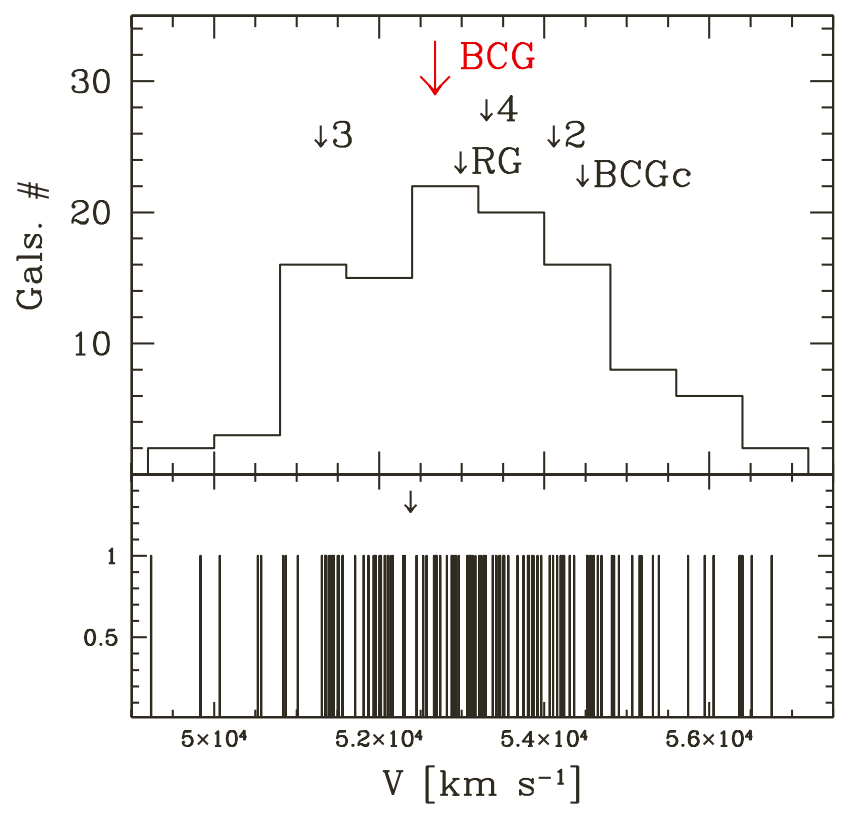

Fig. 7. The 110 galaxies assigned to the cluster. Upper panel: velocity distribution. The arrows indicate the velocities of the BCG, other brightest galaxies, and RG. Lower panel: stripe density plot where the arrow indicates the position of the significant gap.

We then investigate the presence of gaps in the velocity distribution. We follow the weighted gap analysis presented by Beers et al. (1991, 1992; ROSTAT software). We look for normalized gaps larger than 2.25 since in random draws of a Gaussian distribution they arise at most in about $3 \%$ of the cases, independent of the sample size (Wainer \& Schacht 1978). We detect one significant gap (at the $97 \%$ c.l.), which divide the cluster into two groups of 36 and 70 galaxies from low to high velocities (hereafter GV1 and GV2, see Fig. 7). The BCG3 is assigned to the GV1 peak. Other bright galaxies (BCG, BCG2, BCG4, and $\mathrm{BCGc}$ ) are all assigned to the GV2 peak.

To detect subsets in the velocity distribution, we then resort to the Kaye's mixture model (KMM) test (Ashman et al. 1994). The KMM algorithm fits a user-specified number of Gaussian distributions to a dataset and assesses the improvement of that fit over a single Gaussian. In addition, it provides the maximumlikelihood estimate of the unknown n-mode Gaussians and assigns objects into groups. The KMM test is more appropriate in situations where theoretical and/or empirical arguments indicate that a Gaussian model is reasonable. The Gaussian is valid for cluster velocity distributions, where gravitational interactions drive the system toward a relaxed configuration with a Gaussian velocity distribution. The KMM test does not find a two-group partition, which provides a significantly more accurate description of the velocity distribution than a single Gaussian.

To search for a possible physical meaning for the two subclusters determined by the detected significant velocity gap, we also compared the spatial galaxy distributions of GV1 and GV2 two by two. We found no difference according to the the 2D Kolmogorov-Smirnov test (Fasano 1987).

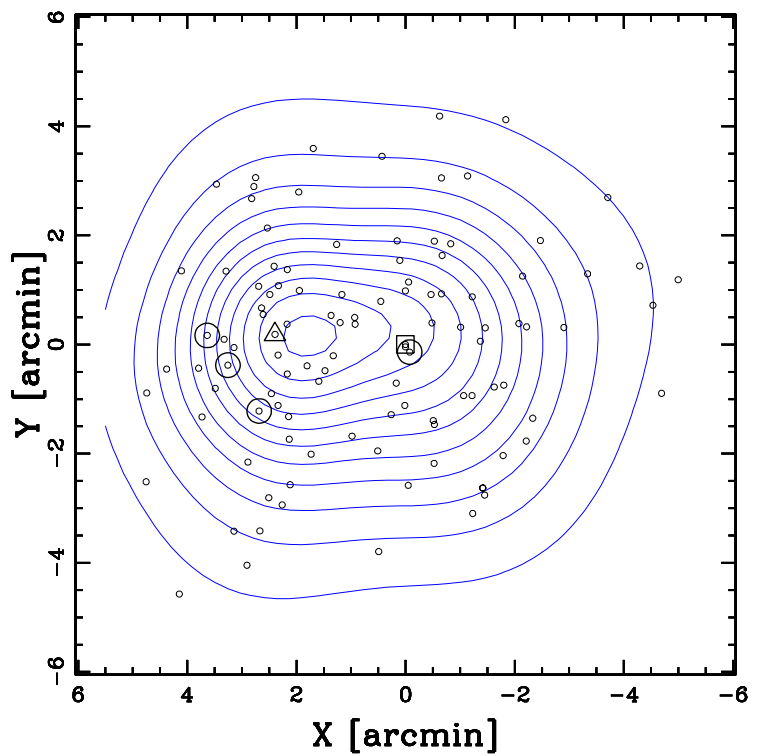

Fig. 8. Spatial distribution on the sky and relative isodensity contour map of spectroscopic cluster members, obtained with the 2D-DEDICA method. The BCG is taken as the cluster center. Large square and circles indicate the location of the BCG and other brightest galaxies. The large triangle indicates RG.

\subsection{Analysis of the $2 D$ galaxy distribution}

By applying the 2D adaptive-kernel method (2D-DEDICA) to the positions of A2254 galaxy members, we found one peak that is $\sim 2^{\prime}$ east of the position of the BCG, and the isodensity contours design a east-west elongated structure (Fig. 8).

Our spectroscopic data do not cover the entire cluster field and are affected by magnitude incompleteness. To overcome these problems, we used our photometric data samples, which cover a larger spatial region. The INT sample has the advantage of having photometry available in three magnitude bands, while the Subaru sample allows us to extend our analysis to fainter galaxies.

When more than two colors are available, it is more effective to select galaxies in the color-color space (Goto et al. 2002). In particular, Lu et al. (2009, see their Figs. 6 and 7) show as $\left(r^{\prime}-\right.$ $i^{\prime}$ ) color can effectively eliminate high-redshift galaxies from the red-sequence galaxies at $z=0.2-0.3$ redshift. In our INT photometric catalog we selected likely members on the basis of both $\left(r^{\prime}-i^{\prime}\right.$ vs. $\left.r^{\prime}\right)$ and $\left(g^{\prime}-r^{\prime}\right.$ vs. $\left.r^{\prime}\right)$ color-magnitude relations (hereafter CMRs), which indicate the early-type galaxies locus. To determine the CMR we applied the $2 \sigma$-clipping fitting procedure to the cluster members: we obtain $r^{\prime}-i^{\prime}=0.777-0.021 \times r^{\prime}$ and $g^{\prime}-r^{\prime}=1.497-0.021 \times r^{\prime}$ (see Fig. 9). Out of our photometric catalog we consider likely "red" cluster members to be those objects lying within 0.1 of the CMRs $\left(r^{\prime}-i^{\prime}\right.$ vs. $\left.r^{\prime}\right)$ and $0.15 \mathrm{mag}$ for $\left(g^{\prime}-r^{\prime}\right.$ vs. $\left.r^{\prime}\right)$. The selected magnitude intervals are chosen with different widths to follow the different amplitudes of the scatter in the two relations: 0.1 and $0.15 \mathrm{mag}$ are round values roughly corresponding to $\sim 1.3$ times the error associated to the fitted intercepts. The difference in the scatter between the two above CMRs is quite expected: for instance, using SDSS data, Goto et al. (2002) compute a scatter of 0.040 and 0.081 for the two above relations.

As a further check, we associated the galaxy type producing the highest value of $\mathcal{R}$ to each galaxy in the RVSAO procedure 


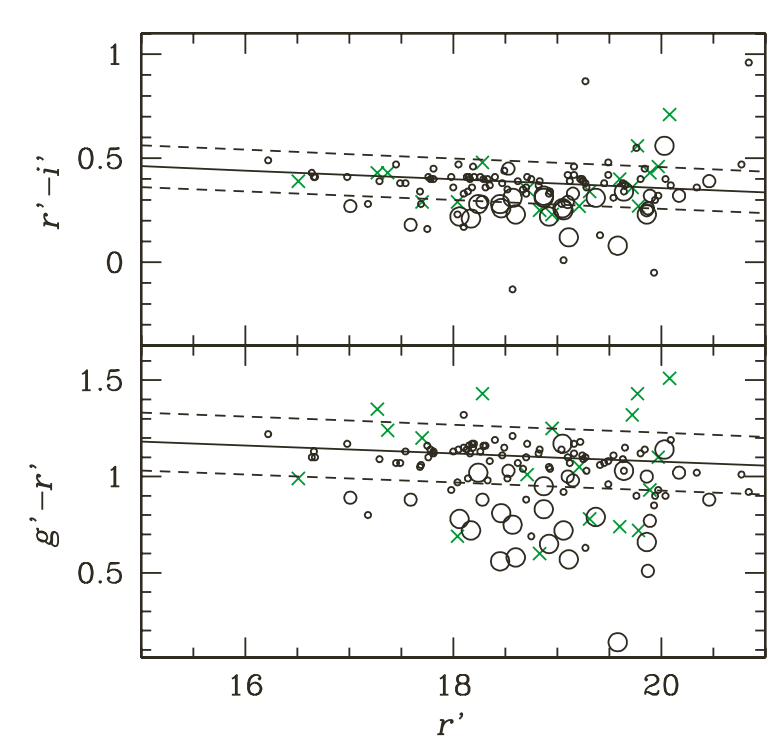

Fig. 9. Upper panel: INT $r^{\prime}-i^{\prime}$ vs. $r^{\prime}$ diagram for galaxies with available spectroscopy. Black circles and green crosses indicate member and nonmember galaxies. For member galaxies large/middle/small circles indicate late/middle/early type galaxies. The solid line gives the CMR determined on member galaxies; the dashed lines are drawn at \pm 0.1 mag from this value. Lower panel: INT $g^{\prime}-r^{\prime}$ vs. $r^{\prime}$ diagram for galaxies with available spectroscopy. The dashed lines are drawn at \pm 0.15 mag from the CMR determined for member galaxies.

and divided galaxies in early-type (E, S0), middle-type ( $\mathrm{Sa}$, $\mathrm{Sb}$ ), and late-type (Sc, Irr). The galaxies with $z$ determined via EMSAO are assigned to late-type galaxies. Although these spectral-types do not pretend to be an alternative to a complete spectral analysis, they are useful for showing how the selection through the CMR, in particular here the $\left(g^{\prime}-r^{\prime}\right.$ vs. $\left.r^{\prime}\right)$, is useful for distinguishing between early- and late-type galaxies (see Fig. 9 - lower panel). Figure 9 shows that the selected magnitude intervals seem adequate for selecting early-type galaxies, thus only using good tracers of the cluster substructure (e.g., Lubin et al. 2000) and, above all, avoiding nonmember galaxies that might bias our 2D analysis.

Figure 10 shows the contour map for the 682 likely cluster members having $r^{\prime} \leq 21$. We find two very significant peaks in the eas-west direction, hereafter $\mathrm{E}$ and $\mathrm{W}$ peaks. The $\mathrm{W}$ peak is at $\sim 1^{\prime}$ of the BCG. A third peak lies in the west south-west region, where our photometric data allow to study very external cluster regions (hereafter "ext" peak). Similar results are found for the 780 objects with $r^{\prime} \leq 21.5$.

As for the results with $r^{\prime} \leq 21$, Table 2 lists the number of assigned members: $N_{\mathrm{S}}$ (Col. 2); the peak position (Col. 3); the density (relative to the densest peak), $\rho_{\mathrm{S}}(\mathrm{Col} .4)$; the value of $\chi^{2}$ for each peak, $\chi_{\mathrm{S}}^{2}$ (Col. 5). Ramella et al. (2007) have tested the 2D-DEDICA procedure on Monte Carlo simulations reproducing galaxy clusters. They show that the physical significance (i.e. the significance that takes the noise fluctuations into account) associated to the subclusters depends on the statistical significance of the subcluster (recovered from the $\chi^{2}$ value) and can be computed using simulations. Considering their Eq. (5), the $\chi^{2}$ threshold for a sample of 682 objects is $\chi_{\text {threshold }}^{2}=32$. Thus, all three peaks that we detect are physically significant.

In the Subaru photometric catalog we selected likely members on the basis of the $\left(V_{\mathrm{obs}}-i_{\mathrm{obs}}^{\prime}\right)$ vs. $i_{\mathrm{obs}}^{\prime}$ diagram. To determine
Table 2. 2D substructure from the INT and SUBARU photometric samples.

\begin{tabular}{|c|c|c|c|c|}
\hline Subclump & $N_{\mathrm{S}}$ & $\begin{array}{c}\alpha(\mathrm{J} 2000), \delta(\mathrm{J} 2000) \\
\mathrm{h}: \mathrm{m}: \mathrm{s}^{\circ}{ }^{\prime}:^{\prime \prime} \\
\end{array}$ & $\rho_{\mathrm{S}}$ & $\chi_{\mathrm{S}}^{2}$ \\
\hline $\mathrm{E}\left(\mathrm{INT} r^{\prime}<21\right)$ & 164 & $171755.2+194113$ & 1.00 & 65 \\
\hline $\mathrm{W}\left(\mathrm{INT} r^{\prime}<21\right)$ & 214 & $171748.0+194144$ & 0.91 & 55 \\
\hline ext $\left(\right.$ INT $\left.r^{\prime}<21\right)$ & 159 & $171631.4+193448$ & 0.33 & 44 \\
\hline $\mathrm{E}\left(\mathrm{SUB} i^{\prime}<20.5\right)$ & 131 & $171755.9+194103$ & 1.00 & 43 \\
\hline $\mathrm{W}\left(\mathrm{SUB} i^{\prime}<20.5\right)$ & 248 & $171746.8+194107$ & 0.94 & 46 \\
\hline $\operatorname{ext}\left(\right.$ SUB $\left.i^{\prime}<20.5\right)$ & 159 & $171638.0+193533$ & 0.37 & 33 \\
\hline SW $\left(\right.$ SUB $\left.i^{\prime}<21.5\right)$ & 122 & $171747.0+194053$ & 1.00 & 37 \\
\hline $\mathrm{E}\left(\mathrm{SUB} i^{\prime}<21.5\right)$ & 77 & $171756.4+194113$ & 0.73 & 31 \\
\hline $\mathrm{NW}\left(\mathrm{SUB} i^{\prime}<21.5\right)$ & 22 & $171746.0+194210$ & 0.69 & 15 \\
\hline $\operatorname{ext}\left(\operatorname{SUB} i^{\prime}<21.5\right)$ & 54 & $171636.5+193545$ & 0.36 & 19 \\
\hline
\end{tabular}

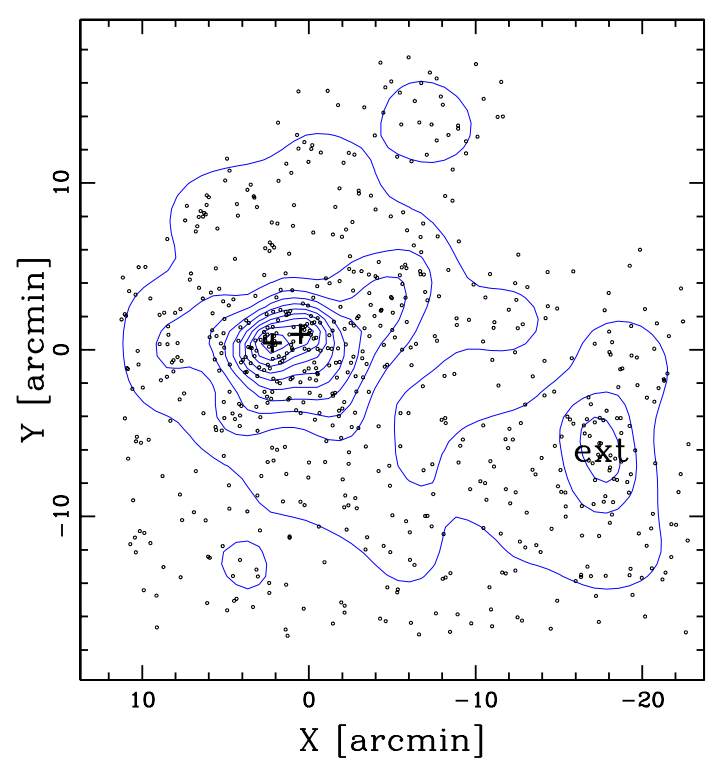

Fig. 10. Spatial distribution on the sky and relative isodensity contour map of INT photometric cluster members with $r^{\prime} \leq 21$, obtained with the 2D-DEDICA method. The two crosses indicate the $\mathrm{E}$ and $\mathrm{W}$ peaks.

the CMR we applied the $2 \sigma$-clipping fitting procedure to the cluster members and obtained $V_{\mathrm{obs}}-i_{\mathrm{obs}}^{\prime}=1.910-0.018 \times i_{\mathrm{obs}}^{\prime}$. Out of our photometric catalog, we consider the objects lying within $0.1 \mathrm{mag}$ of the CMR as likely cluster members (see Fig. 11). We stress that the use of the above color-magnitude relation, which is based on our uncalibrated magnitudes, is valid and only valid within our own catalog. However, we need a (rough) idea of the magnitude correction to understand the depth of our Subaru samples. To this aim, we compared our uncalibrated magnitudes $i_{\text {obs }}^{\prime}$ and magnitudes $i^{\prime}$ of the common objects between the Subaru catalog and the final INT catalog. The visual inspection of the $i_{\text {obs }}^{\prime}-0.1$ vs. $i^{\prime}$ plot, where 0.1 is the Galactic extinction correction, suggests that $i_{\text {obs }}^{\prime} \sim i^{\prime}+0.6$ mag.

Figure 12 shows the 2D-DEDICA results for the sample with $i^{\prime} \leq 20.5$, comparable, for magnitude deepness, to the results from the INT sample with $r^{\prime} \leq 21$. The $i^{\prime} \leq 20.5$ sample confirms the presence of both two density peaks in the galaxy distribution of A2254 and of the external group (841 galaxies in the whole field). However, while the E peak is close to the INT E peak, the $\mathrm{W}$ peak is closer to the BCG position than the INT W peak and both $\mathrm{E}$ and $\mathrm{W}$ structures are someway elongated in the north-south direction. These hints for a more complex structure 


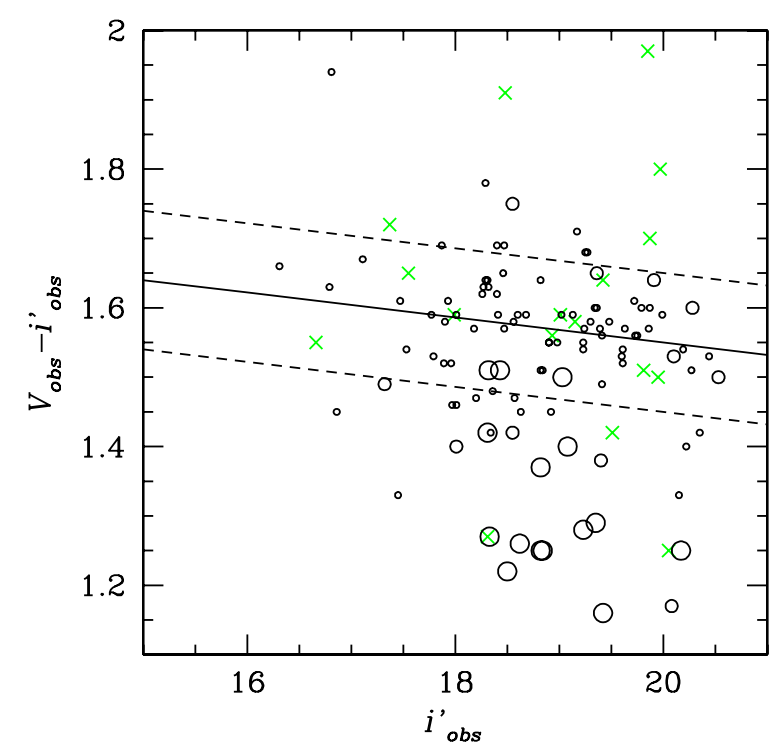

Fig. 11. Subaru $V_{\mathrm{obs}}-i_{\mathrm{obs}}^{\prime}$ vs. $i_{\mathrm{obs}}^{\prime}$ diagram for galaxies with available spectroscopy. Black circles and green crosses indicate member and nonmember galaxies. For member galaxies, large/middle/small circles indicate late/middle/early type galaxies. The solid line gives the CMR determined on member galaxies; the dashed lines are drawn at \pm 0.1 mag from this value.

are reinforced by the analysis of the $i^{\prime} \leq 21.5$ Subaru sample (Fig. 12; 1257 galaxies in the whole field) where the W structure is shown to be bimodal (NW and SW peaks in Table 2). Out of the many peaks obtained through the 2D-DEDICA in the $i^{\prime} \leq 21.5$ Subaru sample, Table 2 lists the results for the three densest peaks and the external peak for comparison. Since in this case $\chi_{\text {threshold }}^{2} \sim 40$, we cannot formally discard the possibility of noise fluctuations, but Fig. 2 of Ramella et al. (2007) suggests a low probability $(\sim 10 \%)$ of noise contamination when the $\chi^{2}$ is about half of $\chi_{\text {threshold }}^{2}$. We do not show results for deeper Subaru samples since, working on galaxies in magnitudes bins, one realizes that the distribution of fainter galaxies is no longer centered on the cluster position, a likely sign of contamination by the background large-scale structure.

The complex structure detected by the 2D-DEDICA method is confirmed by the Voronoi tessellation and percolation (VTP) technique (e.g. Ramella et al. 2001; Barrena et al. 2005). This technique is not parametric and does not smooth the data. As a consequence, it identifies galaxy structures irrespective of their shapes. For our purposes we run VTP on the same Subaru sample of 1257 likely members with $i^{\prime}<21.5$. The result of applying VTP is shown in Fig. 13. VTP is run four times by adopting four detection thresholds: galaxies identified as belonging to structures at $95 \%, 98 \%, 99 \%$, and $99.9 \%$ c.ls. VTP confirms the elongation of the $\mathrm{E}$ and $\mathrm{W}$ structures in the north-south direction. In particular, the W structure is clearly bimodal, and there are clues of a bimodality of the E structure, too. Far from the cluster center, $17^{\prime}$ west south-west, VTP also confirms the existence of the ext peak.

Interestingly, the ext peak can be successfully identified with the cluster NSC J171627+193456 at $z_{\text {phot }} \sim 0.194$, discovered by Gal et al. (2003, based on the galaxy catalogs from the digitized Second Palomar Observatory Sky Survey). However, the absence of spectroscopic $z$ in the region of this system does not allow inferring whether it is a bound companion cluster in the

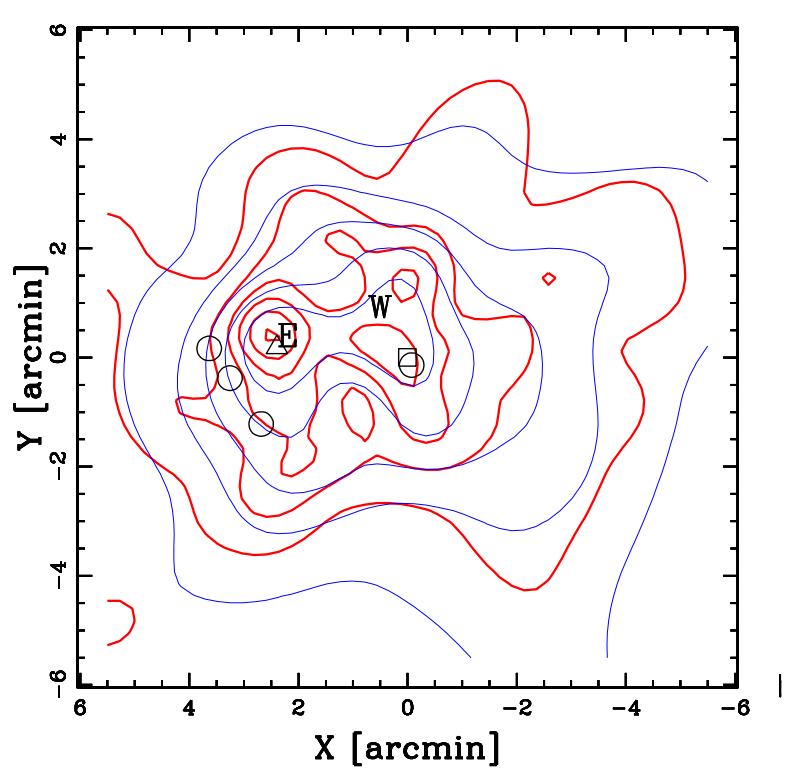

Fig. 12. Spatial distribution on the sky and relative isodensity contour map of Subaru photometric cluster members with $i^{\prime} \leq 20.5$ and with $i^{\prime} \leq 21.5$ (thin/blue and thick/red contours, respectively). Labels E and $\mathrm{W}$ indicate the peaks as detected by DEDICA in the INT sample (see Table 2). The BCG and other interesting galaxies are indicated as in Fig. 8.

outskirts of A2254 (projected at $\sim 3 h_{70}^{-1} \mathrm{Mpc}$ from the cluster center).

\section{5. $3 D$ analysis}

\subsubsection{Significance of 3D substructure}

The existence of correlations between positions and velocities of cluster galaxies is a characteristic of true substructures. Here we use different approaches to analyze the structure of A2254 combining position and velocity information. We find no evidence for a significant velocity gradient (see, e.g., den Hartog \& Katgert 1996; and Girardi et al. 1996, for the details of the method).

To check for the presence of a substructure, we combined velocity and position information by computing the $\Delta$-statistics devised by Dressler \& Schectman (1988, hereafter DS-test), which is recommended by Pinkney et al. (1996) as the most sensitive 3D test. For each galaxy, the deviation $\delta$ is defined as $\delta_{i}^{2}=\left[\left(N_{\mathrm{nn}}+1\right) / \sigma_{\mathrm{V}}^{2}\right]\left[\left(\overline{\mathrm{V}_{1}}-\bar{V}\right)^{2}+\left(\sigma_{\mathrm{V}, 1}-\sigma_{V}\right)^{2}\right]$, where the subscript "l" denotes the local quantities computed over the $N_{\mathrm{nn}}=10$ neighbors of the galaxy, and $\Delta$ is the sum of the $\delta$ of the individual $N$ galaxies that gives the cumulative deviation of the local kinematical parameters (mean velocity and velocity dispersion) from the global cluster parameters (here $N=110$ ). The significance of $\Delta$, i.e. of substructure, is checked by running 1000 Monte Carlo simulations, randomly shuffling the galaxy velocities. We find no significant sign of any substructure.

Following Pinkney et al. (1996; see also Ferrari et al. 2003), we applied two more classical 3D tests: the $\epsilon$-test (Bird 1993) based on the projected mass estimator and the centroid shift or $\alpha$-test (West \& Bothun 1990). The details of these tests can be found in the above papers. We only point out that we considered ten neighbors for each galaxy, and we used the above Monte Carlo simulations to compute the substructure 


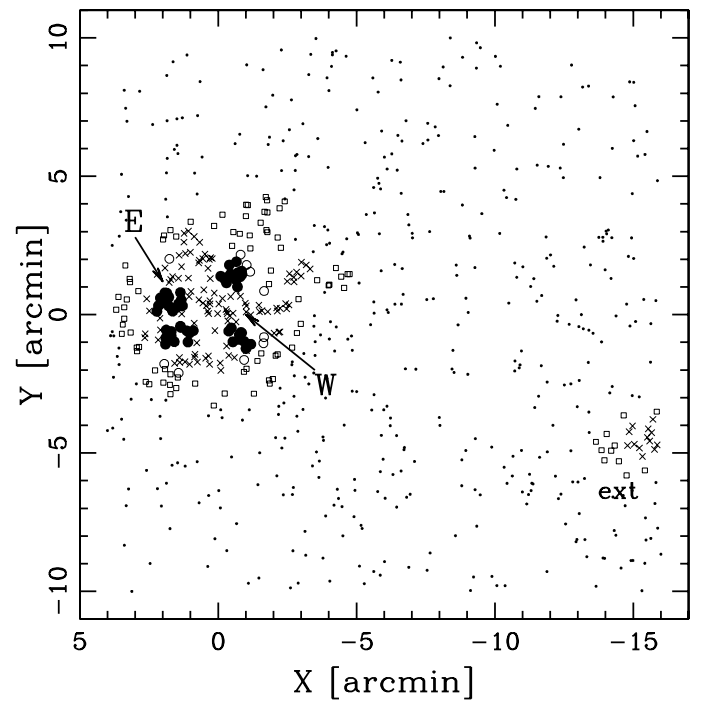

Fig. 13. Galaxies belonging to structures as detected by the Voronoi Tessellation and Percolation technique. The algorithm is run on the sample of likely members with $i^{\prime}<21.5$ extracted from the Subaru photometric catalog (see text). Open squares, open circles, crosses, and solid circles indicate galaxies in structures at the 95\%, 98\%, 99\%, and $99.9 \%$ c.ls., respectively.

significance. In both cases we did not find any evidence of significant substructure.

We also considered two kinematical estimators alternative to the $\delta$ parameter of the DS-test, i.e. we considered separately the contribution of the local mean $\left.\delta_{V}^{2}=\left[\left(N_{\mathrm{nn}}+1\right) / \sigma_{V}^{2}\right]\left(\overline{V_{l}}-\bar{V}\right)^{2}\right]$ and dispersion $\left.\delta_{\mathrm{s}}^{2}=\left[\left(N_{\mathrm{nn}}+1\right) / \sigma_{V}^{2}\right]\left(\sigma_{V, 1}-\sigma_{V}\right)^{2}\right]$ (see, e.g., Girardi et al. 1997; Ferrari et al. 2003). When considering the $\delta_{\mathrm{s}}$ estimator, we found evidence of a peculiar local velocity dispersion at the $97 \%$ c.l. Figure 14 (lower panel) plots the distribution on the sky of all galaxies showing the deviations of the local velocity dispersion from the global cluster value. The two largest deviations are obtained for the subgroups associated to two galaxies just south of the BCG for which $\sigma_{V, l} \sim 400 \mathrm{~km} \mathrm{~s}^{-1}$. Other deviations interest the east region characterized by high values for the velocity dispersion. To investigate more closely we repeated the DS-test, increasing the number of neighbors. As for the $\delta_{\mathrm{s}}$ indicator, we obtain high c.l. values for peculiarity up to a $99.99 \%$ c.l. in the $N_{\mathrm{nn}}=25-50$ range (see Fig. 15 - upper panel). This result suggests that the peculiarity pertains to a large part of the cluster. To determine how many galaxies are involved in the substructure, we resort to the technique developed by Biviano et al. (2002), who use the individual $\delta_{i}$-values of the DS method. The comparison of the $\delta_{\mathrm{s}, i}$-values of all 1000 Monte Carlo simulations and the observed values shows how many galaxies are involved in the structures with peculiar kinematics (Fig. 15 - lower panel).

\subsubsection{Identifying subclusters in the east region}

Comparing the two subsamples obtained by a rough division of A2254 (e.g., the eastern and the western subsamples with respect to the position of the BCG), we find that the eastern sample is characterized by a higher value of $\sigma_{V}$ (see Table 3 and Fig. $16-$ upper panel). The F-test confirms that the velocity dispersions of the two samples differ at the $99.4 \%$ c.l.; no difference is shown

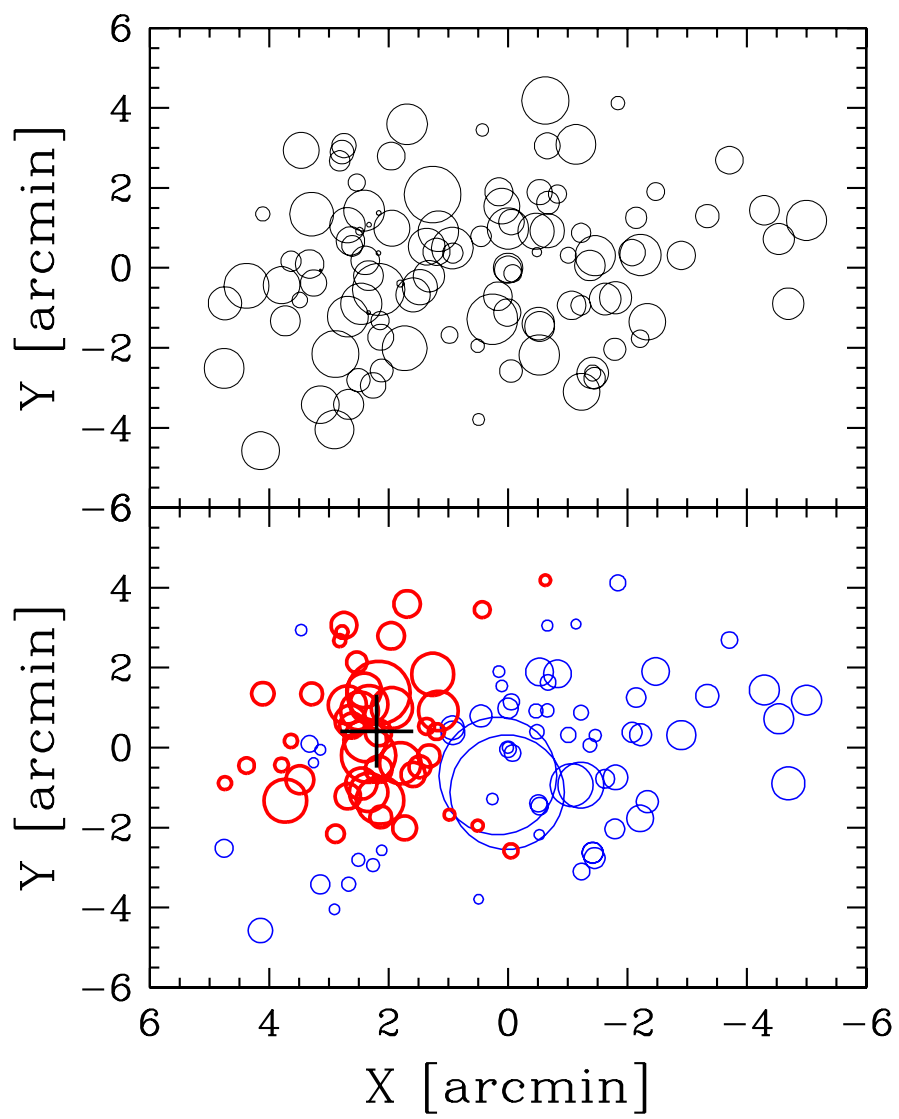

Fig. 14. Spatial distribution of the 110 cluster members, each marked by a circle. The plot is centered on the cluster center. Upper panel: the cluster velocity field: the higher the circle, the larger is the galaxy velocity. Lower panel: the result of the (modified) DS-test: the larger the circle, the greater is the deviation $\delta_{\mathrm{s}, i}$ of the local velocity dispersion from the global velocity dispersion. Thin/blue and thick/red circles show where the local velocity dispersion is smaller or larger than the global value. The cross indicates the position of the E peak as detected in the $2 \mathrm{D}$ analysis (INT sample).

between the mean velocities according to the means test (see, e.g., Press et al. 1992, for these tests).

The lower panel of Fig. 15 shows as the region with high $\sigma_{V, l}$ values is centered roughly on the E peak as determined in the 2D analysis. We selected the galaxies within a circular region centered on the position of the E peak. Using a radius of $1.5^{\prime}$, comparable to the distance between $\mathrm{E}$ peak and $\mathrm{W}$ peaks, we obtained a subsample of 27 galaxies, the E1.5 sample, characterized by a high value of $\sigma_{V} \sim 1700 \mathrm{~km} \mathrm{~s}^{-1}$. When applying the 1D-DEDICA method to this subsample we detected two significant peaks with 21 galaxies at $\sim 52500$ and 6 at $56300 \mathrm{~km} \mathrm{~s}^{-1}$ (see E1.5LV and E1.5HV in Table 3). Figure 16 - lower panel - shows the results of the 1D-DEDICA method for the E1.5 sample, as well as for other two samples defined by a larger and a smaller radius $\left(2^{\prime}\right.$ and $\left.1^{\prime}\right)$. The choice of a large radius gives one-(asymmetric) peak in the galaxy density distribution in the velocity space, while too small a radius gives a list of insignificant peaks owing to the small-sample statistics involved. 
Table 3. Kinematical properties of the whole system and galaxy subsystems.

\begin{tabular}{|c|c|c|c|c|c|c|c|}
\hline \multicolumn{4}{|c|}{ Substructure results } & \multicolumn{4}{|c|}{ 3D KMM } \\
\hline System & $N_{\mathrm{g}}$ & $\begin{array}{c}\langle v\rangle \\
\mathrm{km} \mathrm{s}^{-1}\end{array}$ & $\begin{array}{c}\sigma_{V} \\
\mathrm{~km} \mathrm{~s}^{-1}\end{array}$ & Interpretation & $N_{\mathrm{g}, \mathrm{KMM}}$ & $\begin{array}{l}\langle v\rangle_{\mathrm{KMM}} \\
\mathrm{km} \mathrm{s}^{-1}\end{array}$ & $\begin{array}{l}\sigma_{v, \mathrm{KMM}} \\
\mathrm{km} \mathrm{s}^{-1}\end{array}$ \\
\hline Whole system & 110 & $53128 \pm 128$ & $1340_{-84}^{+101}$ & - & - & - & - \\
\hline Eastern system & 58 & $53098 \pm 208$ & $1576_{-104}^{+168}$ & contaminated main & - & - & - \\
\hline Western system & 52 & $53186 \pm 150$ & $1073_{-92}^{+118}$ & uncontaminated main & - & - & - \\
\hline E1.5HV & 6 & $56310 \pm 226$ & $245_{-180}^{+96}$ & high-vel. E group & 7 & $56414 \pm 116$ & $370_{-181}^{+291}$ \\
\hline E1.5LV & 21 & $52463 \pm 341$ & $1000_{-102}^{+220}$ & main & 103 & $52968 \pm 119$ & $1180_{-67}^{+88}$ \\
\hline HT2 & 7 & $56355 \pm 244$ & $232_{-104}^{+68}$ & high-vel. E group & 8 & $56341 \pm 115$ & $469_{-255}^{+187}$ \\
\hline HT1 & 96 & $52980 \pm 108$ & $1053_{-66}^{+59}$ & (substructured) main & - & - & - \\
\hline HT12 & 8 & $51414 \pm 159$ & $421_{-91}^{+66}$ & low vel. W main & 35 & $51840 \pm 100$ & $889_{-63}^{+162}$ \\
\hline HT11 & 42 & $53299 \pm 188$ & $739_{-55}^{+81}$ & principal main & 67 & $53550 \pm 153$ & $939_{-69}^{+86}$ \\
\hline DED1 & 3 & $56322 \pm 139$ & $197^{a}$ & high-vel. E group & 4 & $56422 \pm 109$ & $167_{-132}^{+70}$ \\
\hline DED2 & 9 & $54313 \pm 213$ & $579_{-80}^{+118}$ & NW group & 27 & $54246 \pm 130$ & $663_{-68}^{+98}$ \\
\hline DED3 & 12 & $53151 \pm 362$ & $1171_{-134}^{+248}$ & E group & 26 & $53385 \pm 320$ & $1591_{-193}^{+329}$ \\
\hline DED4 & 30 & $53054 \pm 177$ & $950_{-98}^{+141}$ & SW group & 46 & $52168 \pm 114$ & $763_{-66}^{+84}$ \\
\hline DED5 & 4 & $51798 \pm 293$ & $448_{-46}^{+21}$ & low vel. SE group & 7 & $53238 \pm 429$ & $1000_{-59}^{+407}$ \\
\hline
\end{tabular}

Notes. ${ }^{(a)}$ Standard velocity dispersion estimate.

\subsubsection{Identifying subclusters with the Htree method}

We resort to the method devised by Serna \& Gerbal (1996, hereafter Htree-method; see, e.g., Durret et al. 2010 for a recent application). This method uses a hierarchical clustering analysis to determine the relationship between galaxies according to their relative binding energies. The method assumes a constant value for the mass-to-light ratio of galaxies, and Serna \& Gerbal suggest a value comparable to that of clusters. Here we take a value of $M / L_{r}=150 h_{70} M_{\odot} / L_{\odot}$, as suggested by broad statistical studies (e.g., Girardi et al. 2000; Popesso et al. 2005). The (gross) results are quite robust against the choice of the value of $M / L_{r}$.

Figure 17 shows the resulting dendogram, where the total energy appears horizontally. Galaxy pairs and subgroups of galaxies then appear with a lower total energy. Starting from the shallower part of the energy levels (righthand part of the dendogram) we find HT1, which we interpret as the main system, and the group HT2. HT2 is a high-velocity eastern group that contains all members of E1.5HV. HT1 is a "substructured" main system formed by HT11, which has the BCG in its potential well, and HT12, i.e. a few low velocity galaxies located in the west region (in particular, the BCG3). However, at the bottom of the binding energy of HT11 we find HT1111, which is not a core in the classical sense. In fact, HT1111 contains HT11111 and HT11112. HT11112 is formed by three galaxies close to the cluster center: the BCG and BCGc pair, and a small compact galaxy located between them. HT11111 is close to the E peak and is formed by four galaxies, among which the BCG2 and BCG4.

\subsubsection{Identifying subclusters with the 3D-DEDICA method}

Finally, we apply the 3D-DEDICA method (Bardelli et al. 1998). Table 3 lists the five most significant ( $294 \%$ c.1.) groups (DED1DED5) composed of at least three galaxies. The picture revealed by the 3D-DEDICA test is that of a very complex structure, with three subclusters in the east and two in the west region.

Figure 18 (left col.) shows the spatial distribution of the groups detected by the above methods, and Table 3 lists the kinematical properties: the number of assigned members, $N_{\mathrm{g}}$
(Col. 2); the mean velocity, $\langle v\rangle$ (Col. 3); the velocity dispersion with bootstrap errors, $\sigma_{V}$ (Col. 4); a short comment summarizing our interpretation (Col. 5). The rest of the properties refer to the corresponding KMM subclusters, see below (Cols. 6-8).

\subsubsection{D-KMM method}

We use the above results to apply the full 3D-KMM method, which, starting from the above seeds, allow us to assign all galaxies to subclusters. Using E1.5LV and E1.5HV as seeds for a initial two-group partition, we find that a partition of 103 and 7 galaxies is a significantly more accurate description of the 3D galaxy distribution than a single 3D Gaussian (at the $99.7 \%$ c.1.). We note that the KMM-E1.5LV subcluster. i.e. the main system, has a significant weighted gap in its velocity distribution (as in the whole sample, see Sect. 3.3) but does not show any trace of peculiar kinematics according to the DS-test. Using HT1, HT2, and HT1111 as initial seeds, the 3D-KMM converges to a three-group partition significant at the $97.5 \%$ c.l. Finally, using DED1-DED5 groups as initial seeds we find the 3D-KMM test converges to a five-group partition significant at the $99.9 \%$ c.l. Figure 18 (right col.) shows the spatial distribution of the obtained partitions.

\section{X-ray morphological and spectral analysis}

\subsection{Observation and data reduction}

A2254 was observed by XMM-Newton on August 26, 2009. The data were reduced with SAS v10.0 using the tasks emchain and epchain. We only considered event patterns $0-12$ for MOS and 0 for $\mathrm{pn}$, and the data were cleaned using the standard procedures for bright pixels and hot columns removal (by applying the expression FLAG $==0$ ) and pn out-of-time correction. The energy scale of the pn over the whole spectral bandpass was improved further with the task epreject. Periods of high background event counts due to soft protons were filtered using a threshold cut method: we performed a Gaussian fit to the peak of the histogram of the light curve and excluded the time bins where the count rate lies more than $3 \sigma$ away from the mean. 

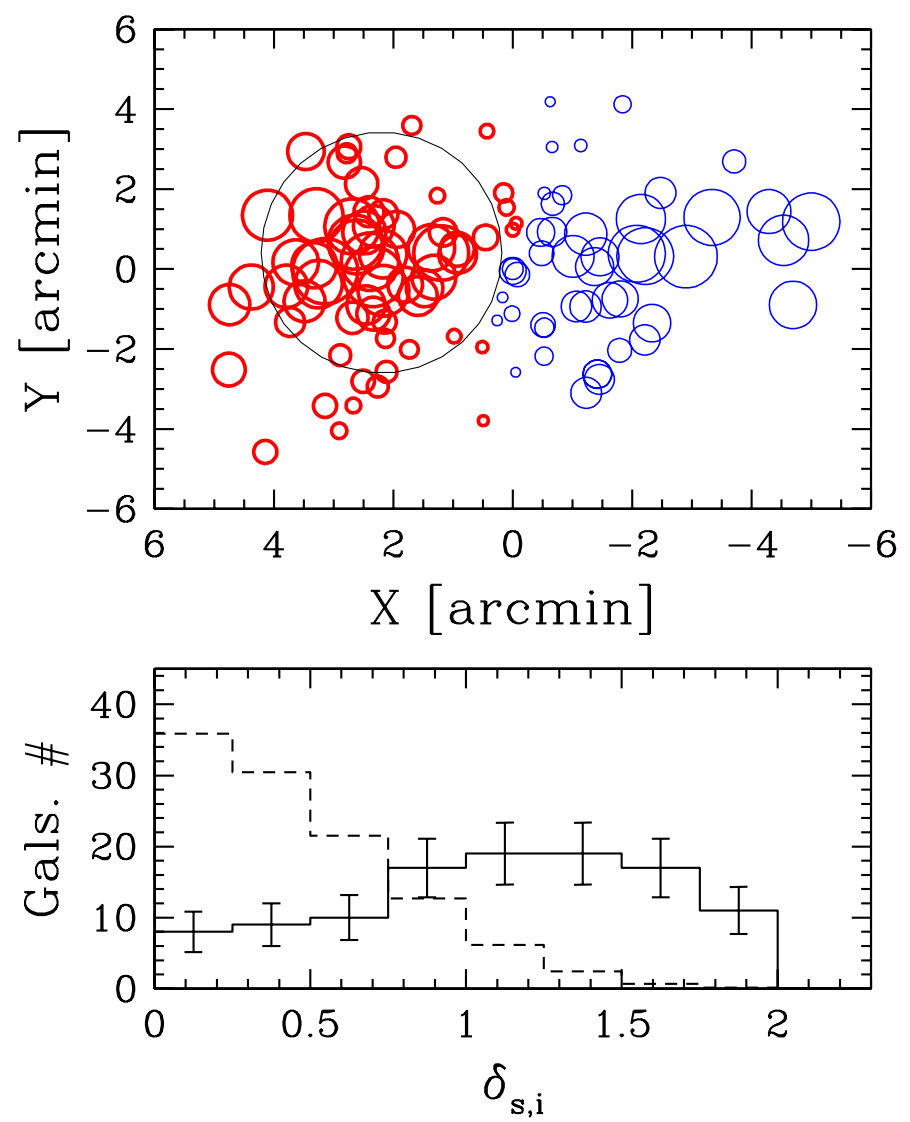

Fig. 15. Upper panel: the same as Fig. 14 - lower panel but for the DStest with a larger number of neighbors $\left(N_{\mathrm{nn}}=40\right)$. The large faint circle encloses the region within $1.5^{\prime}$ from the E peak: galaxies inside define the E1.5 sample. Lower panel: the distribution of $\delta_{\mathrm{s}, i}$ deviations in the above DS-test. The solid line represents the observations, the dashed line the distribution for the galaxies of the 1000 simulated clusters, normalized to the observed number.

The light curves were extracted from regions of least source contamination (excising the bright object core in the central $8^{\prime}$ and the point source list from the SOC pipeline, after visual inspection) in two different energy bands: a hard band of $10-12 \mathrm{keV}$ for MOS and 10-13 keV for pn (using 100 s bins) and a wider band, $0.5-10 \mathrm{keV}$ (using 10s bins), as a safety check for possible flares with soft spectra. The flaring periods thus determined were checked further by visual inspection of the light curves, resulting in net exposures of $57.0 \mathrm{ks}, 56.8 \mathrm{ks}$, and $45.1 \mathrm{ks}$ for the MOS1, MOS2, and pn detectors, respectively. Point sources were detected using the task ewavelet in the energy band $0.5-10 \mathrm{keV}$ and checked by eye on images generated for each detector. Detected point sources from all detectors were merged, and the events in the corresponding regions removed from the event list using circular regions of $25^{\prime \prime}$ radius centered at the source position. The area lost by point-source exclusion, CCD gaps, and bad pixels was calculated using a mask image. Redistribution matrix files (RMFs) and ancillary response files (ARFs) were generated with the SAS tasks rmfgen and arfgen, the latter in extended source mode. Appropriate flux-weighting was performed for RMFs, and for ARFs using exposure-corrected images of the source as detector maps (with pixel size of $1^{\prime}$, the minimum scale modeled by arfgen) to sample the variation in emission, following the prescription of Saxton \& Siddiqui (2002). For the background subtraction, we adopted a complete modeling of the various background components as described in detail in

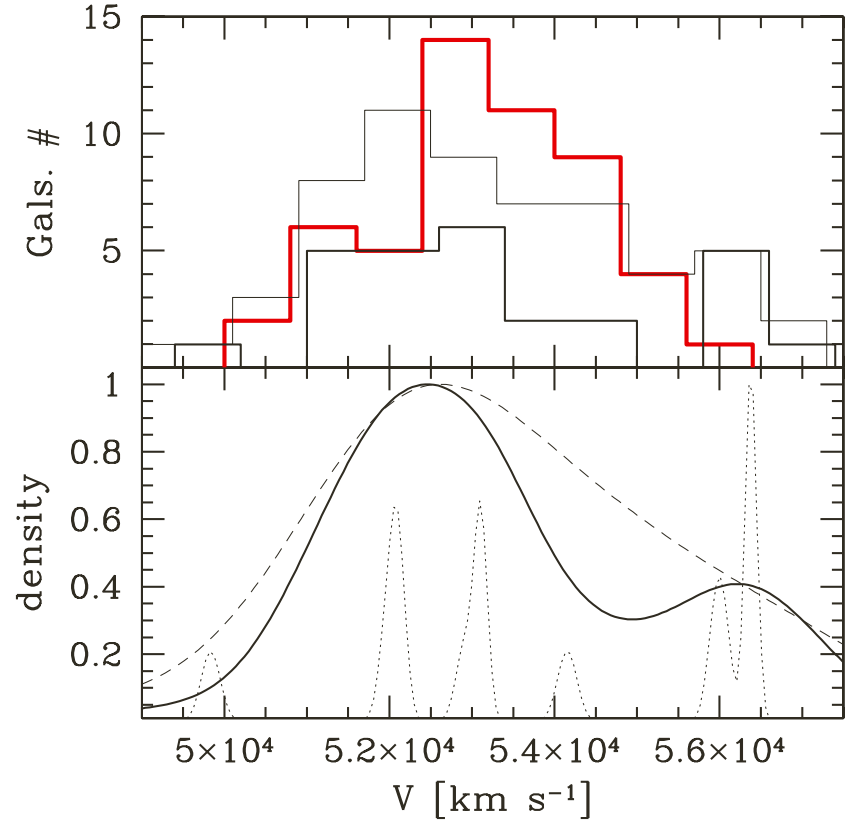

Fig. 16. Upper panel: velocity distributions of the galaxies in the western and eastern samples (thick/red and thin/black lines, respectively). The velocity distribution of a subsample of the eastern sample, i.e. the 27 galaxies within a radius of $1.5^{\prime}$ from the E peak (E1.5 sample), is also shown (black thick line). Lower panel: the galaxy density distribution in the velocity space, as provided by the DEDICA adaptive-kernel reconstruction method for the E1.5 sample (solid line) and for two samples contained within a larger and a smaller radius, E2.0 and E1.0 samples (dashed and dotted lines, respectively). Units on the $y$-axis are normalized to the density of the highest peak.

Gastaldello et al. (2007). We accurately modeled the actual sky background using a region free of cluster emission at $r>9^{\prime}$, where the surface brightness profile in the $0.7-1.2 \mathrm{keV}$ reaches the background level. The spectra of the out-of-field-of-view events of CCDs 4 and 5 of MOS1 showed an anomalously high flux in the soft band (see Kuntz \& Snowden 2008), so they were excluded from our surface brightness and spectral analysis.

\subsection{X-ray image and surface-brightness analysis}

For each MOS detector, we created images in the $0.5-2 \mathrm{keV}$ band and exposure maps at the monochromatic energy of $1 \mathrm{keV}$ and then combined the images into a single exposure-corrected image, smoothed on a scale of $12^{\prime \prime}$, as shown in Fig. 19. We processed the image to remove the point sources using the CIAO tool dmfilth, which replaces photons in the vicinity of each point source with a locally estimated background. We considered only the regions exposed by more than $65 \%$ of the maximum exposure (see Fig. 20) in order to perform the X-ray morphology analysis. We adopt quantitative measures by applying the power ratios technique (Buote \& Tsai 1996), the centroid shifts (Mohr et al. 1993) and the concentration parameter (Santos et al. 2008).

Power ratios derive from multipole expansions of the 2D projected gravitational potential and can be applied directly on $\mathrm{X}$-ray images since under normal circumstances the X-ray emissivity can be used as a proxy for the gravitational potential. Power ratios are computed within a fixed circular aperture, and they can be computed on the centroid of the cluster or on the 


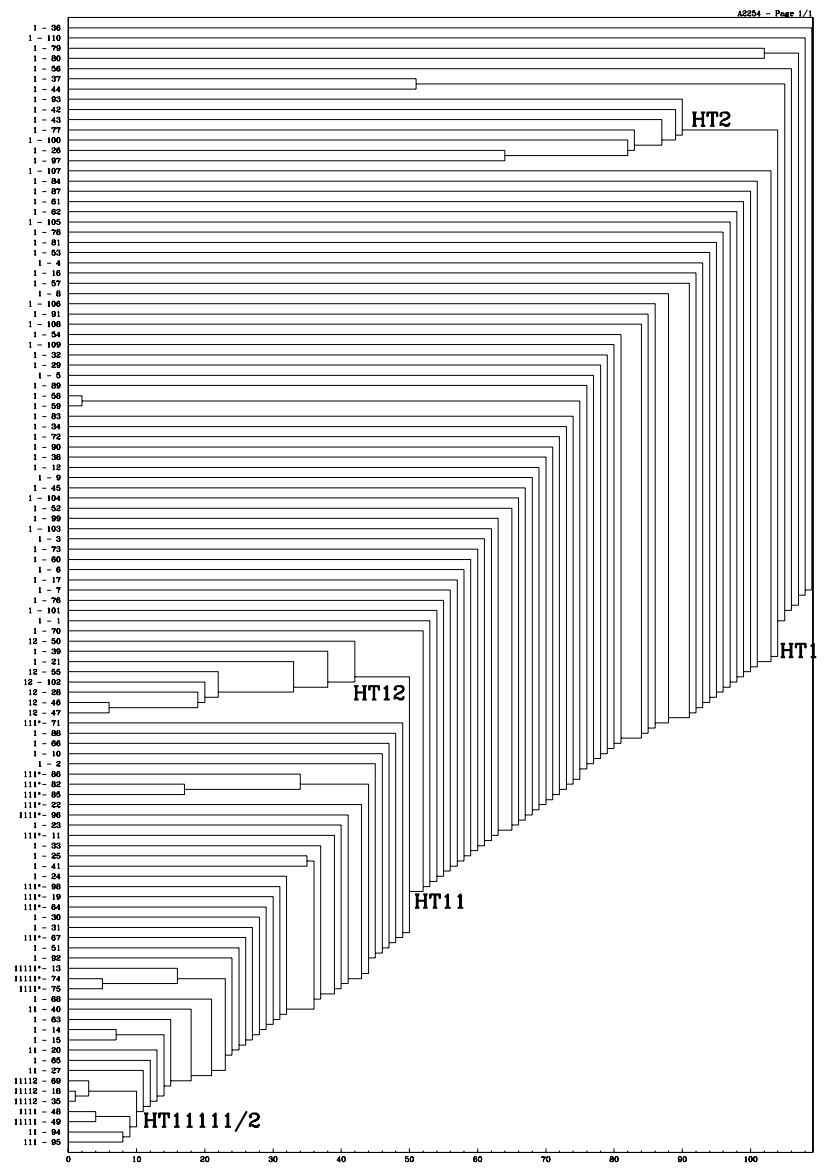

Fig. 17. Dendogram obtained through the Serna \& Gerbal (1996) algorithm. The abscissa is the binding energy (here in arbitrary unit with the deepest negative energy levels on the left), while the catalog numbers of the various member galaxies are shown along the ordinate (IDm in Table 1).

$\mathrm{X}$-ray peak. In the former case, $P_{1} / P_{0}$ is zero, $P_{2} / P_{0}$ gives information on the ellipticity of the cluster, $P_{3} / P_{0}$ is related to a bimodal structure, and $P_{4} / P_{0}$ is sensitive on a small-scale substructure. In the latter case, $P_{1}^{\mathrm{pk}} / P_{0}^{\mathrm{pk}}$ is not zero if the cluster does not exhibit reflection symmetry about two orthogonal axes originating at the peak of the emission, and it is essentially a circularly average centroid shift. We calculate the power ratios within an aperture of $500 \mathrm{kpc}$, given the high signal-to-noise within this radius and for comparison with previous work.

Centroid shifts indicate that the center of mass of the X-ray emitting gas varies with radius. Centroid shifts and power ratios are both capable of identifying highly disturbed systems or systems with significant, well defined substructures (Poole et al. 2006); however, the centroid shifts parameter, $w$, is more sensitive to subtler disturbances. Following the method of O'Hara et al. (2006; see also Poole et al. 2006), the centroid shift is computed in a series of circular apertures centered on the cluster $\mathrm{X}$-ray peak. The radius of the apertures is decreased in steps of $5 \%$ from $500 \mathrm{kpc}$ to $25 \mathrm{kpc}$.

The concentration parameter, $c$, is defined as the ratio of the peak (calculated within $100 \mathrm{kpc}$ ) over the ambient surface brightness (calculated within $500 \mathrm{kpc}$ ). We applied these techniques to the image reported in Fig. 20 and the results are reported in Table 4.

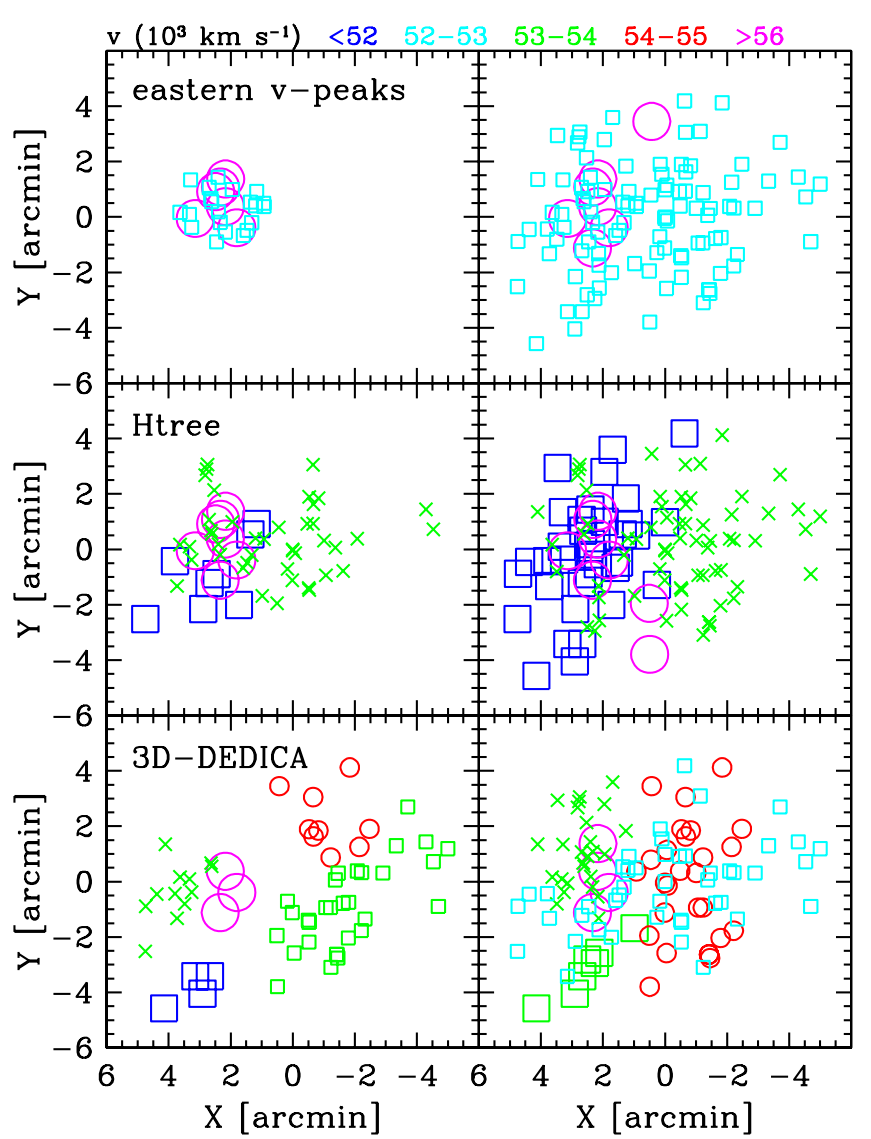

Fig. 18. Spatial distribution on the sky of the galaxies for the subclusters detected by our 3D analysis and the cluster partitions obtained through the 3D-KMM method using them as seeds (left and right panels, respectively). In the left panels symbols refer to different groups. The different colors refer to different mean velocities according to the legend at the top of the figure. In the right panels symbols indicate subclusters found using the respective groups on the left as seeds, and colors refer again to the velocity according to the above legend.

Table 4. Results of the morphological analysis of the X-ray image of A2254 using power ratios, centroid shifts and concentration.

\begin{tabular}{lccccc}
\hline \hline $\begin{array}{l}P_{1}^{\mathrm{pk}} / P_{0}^{\mathrm{pk}} \\
\left(\times 10^{-4}\right)\end{array}$ & $\begin{array}{c}P_{2} / P_{0} \\
\left(\times 10^{-7}\right)\end{array}$ & $\begin{array}{c}P_{3} / P_{0} \\
\left(\times 10^{-7}\right)\end{array}$ & $\begin{array}{c}P_{4} / P_{0} \\
\left(\times 10^{-8}\right)\end{array}$ & $\begin{array}{c}w \\
\left(500 \mathrm{~h}_{70}^{-1} \mathrm{kpc}\right)\end{array}$ & $c$ \\
\hline $1.84 \pm 0.47$ & $4.25 \pm 1.26$ & $2.51 \pm 0.82$ & $1.29 \pm 1.12$ & $0.037 \pm 0.001$ & 0.08 \\
\hline
\end{tabular}

Following Ettori et al. (2010) we extracted the surface brightness profile in the energy band $0.7-1.2 \mathrm{keV}$ to keep the background as low as possible with respect to the source. For this reason, we avoid the intense fluorescent instrumental lines of $\mathrm{Al}(\sim 1.5 \mathrm{keV})$ and $\mathrm{Si}(\sim 1.8 \mathrm{keV})$. We account for the X-ray background by including a constant background component. The data are grouped to have at least 20 counts per bin in order to apply the $\chi^{2}$ statistics. The fitted model is convolved with the $X M M$-Newton PSF. The joint best-fit $\beta$-model has a core radius of $r_{\mathrm{c}}=(507 \pm 36) h_{70}^{-1} \mathrm{kpc}\left(169^{\prime \prime} \pm 12^{\prime \prime}\right)$ and $\beta=0.95 \pm 0.06$ for a $\chi^{2} /$ d.o.f. $=215 / 179$ (see Fig. 21). 


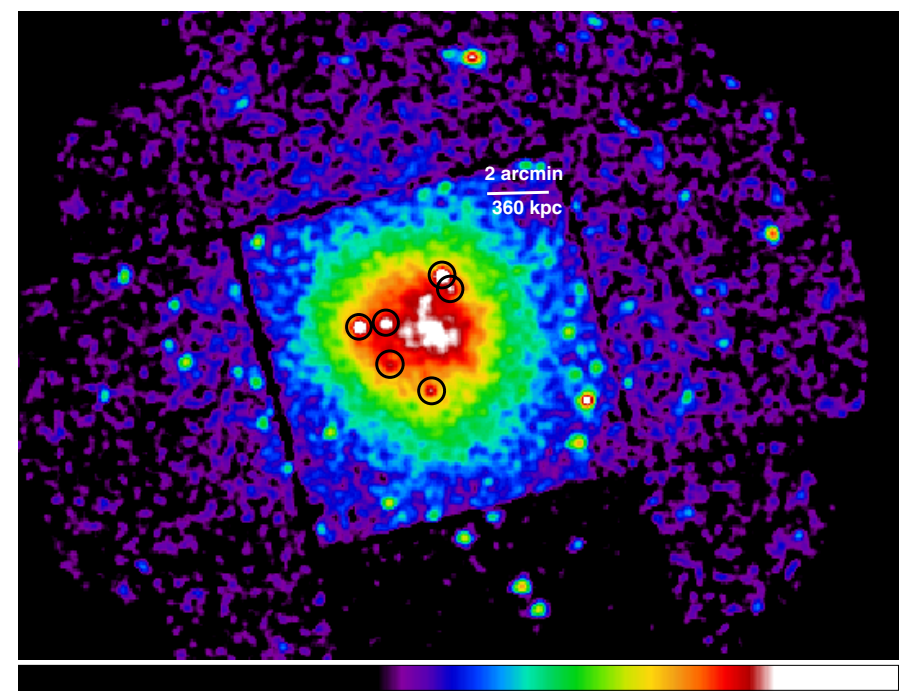

Fig. 19. Mosaic of the MOS 1 and MOS2 images in the $0.5-2 \mathrm{keV}$ energy band smoothed on a $12^{\prime \prime}$ scale. The image was divided by the summed exposure maps to correct for exposure variations. The detected point sources within the central regions of the cluster discussed in the text are highlighted by black circles.

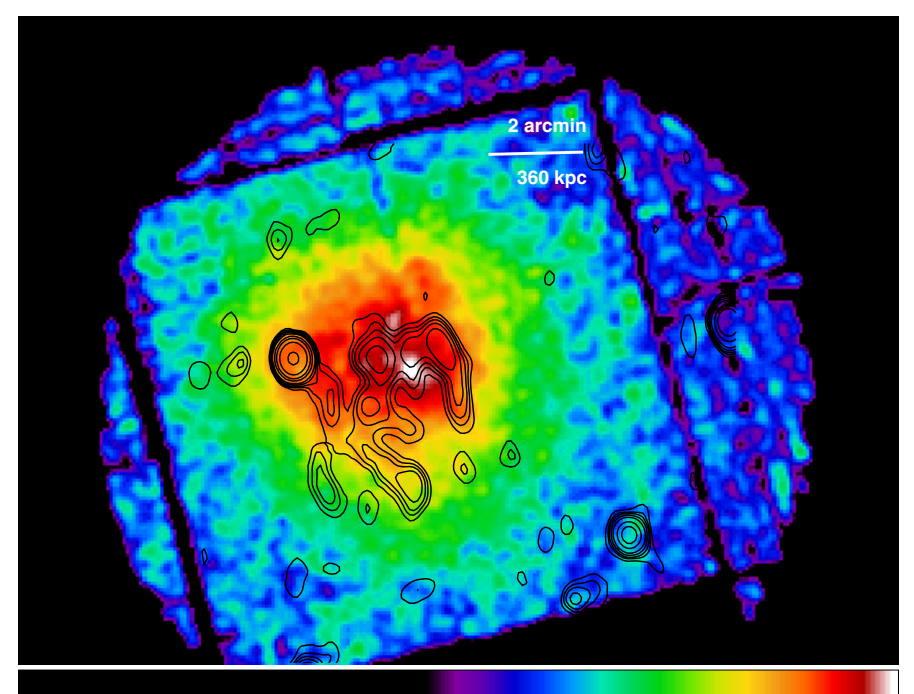

Fig. 20. X-ray image of the diffuse emission of the A2254 cluster where point sources were removed and replaced by a local estimate of the surrounding emission and only regions exposed by more than $65 \%$ of the maximum exposure is considered. The contour levels of the radio VLA image (Govoni et al. 2001b) are superimposed, too.

\subsection{Spectral analysis}

We extracted spectra for each detector in five concentric circular annuli located at the the peak of the X-ray emission with bounding radii $0^{\prime}-0.5^{\prime}, 0.5^{\prime}-1.5^{\prime}, 1.5^{\prime}-2.5^{\prime}, 2.5^{\prime}-3.5^{\prime}$, and $3.5^{\prime}-4.5^{\prime}$. The spectra from the three detectors were re-binned to ensure a signal-to-noise ratio of at least three and a minimum 20 counts per bin (necessary for the validity of the $\chi^{2}$ minimization method). We fit the spectra with an APEC thermal plasma modified by Galactic absorption fixed at $4.7 \times 10^{20} \mathrm{~cm}^{-2}$ (Kalberla et al. 2005) to each annulus. A correction file derived from the background modeling is used in XSPEC as in Gastaldello et al. (2007). The free parameters are temperature, normalization (proportional to emission measure), and the metallicity, with the

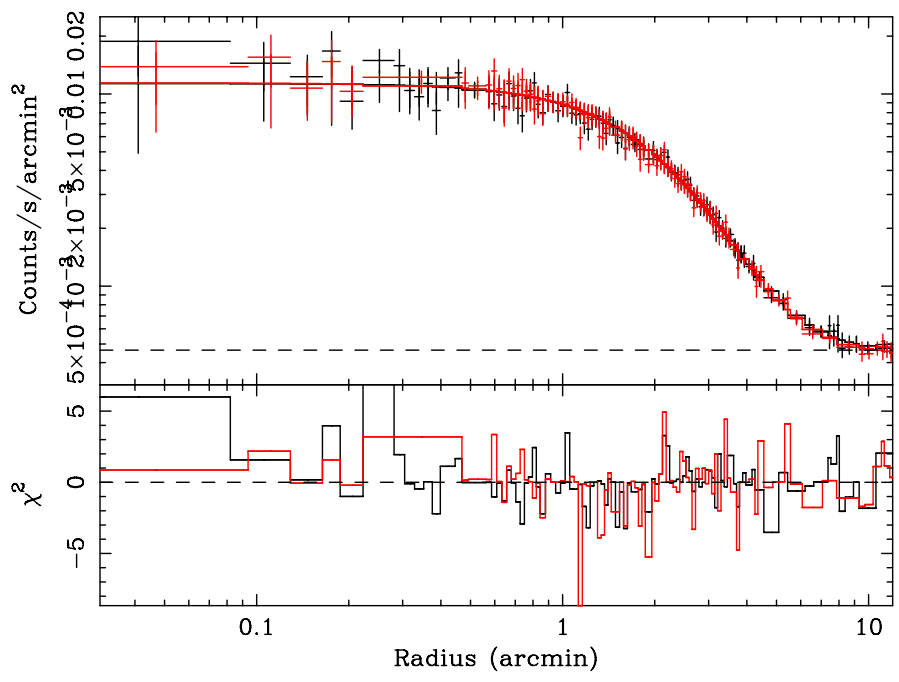

Fig. 21. Surface brightness profile of the X-ray emission of A2254. Data from MOS1 are plotted in black and from MOS2 in red. The best-fit $\beta$ model and ratio of data over the model are also shown. The background level is shown as the black dashed line in the lower panel.

solar abundance units of Grevesse \& Sauval (1998). The spectra are adequately fitted by the model, with reduced $\chi^{2}$ ranging from 1.06 in the inner annulus to 1.5 in the outermost annulus. The azimuthally averaged, projected temperature and abundance profile thus obtained are shown in Fig. 22. The temperature profile is rather flat, and in particular there is no evidence of any decrease in the core, as found in non-cool core clusters. We calculated the mean temperature of the cluster as the temperature obtained with a spectral fit in the region $0.05 R_{180}<r<0.2 R_{180}$, where $R_{180}=1780(k T / 5 \mathrm{keV})^{1 / 2} h(z)^{-1} h_{70}^{-1} \mathrm{kpc}$ (Arnaud et al. 2005 , with $\left.h(z)=\left(\Omega_{\mathrm{M}}(1+z)^{3}+\Omega_{\Lambda}\right)^{1 / 2}\right)$ using an iterative procedure to calculate $k T$ and $R_{180}$ (Rossetti \& Molendi 2010). We find $k T=6.38 \pm 0.25 \mathrm{keV}$ and $R_{180}=2.08 h_{70}^{-1} \mathrm{Mpc}$. The value of $k T$ thus estimated within the $0.05 R_{180}<r<0.2 R_{180}$ region, hereafter $k T_{\text {OUT }}$, is a good proxy for the global temperature (see Fig. 4 of Leccardi \& Molendi 2008, where $k T_{\mathrm{M}}$ is the global temperature). As for A2254, Fig. 22 shows as the measure of $k T$ already has large uncertainties around $0.4 R_{180}$.

To characterize temperature and emission measure gradients, we compared the central to a global value of the pseudo-entropy measure. Following Leccardi et al. (2010), we computed the pseudo-entropy ratio $\mathcal{E}=\left(k T_{\mathrm{IN}} / k T_{\mathrm{OUT}}\right) \times\left(E M_{\mathrm{IN}} / E M_{\mathrm{OUT}}\right)^{-1 / 3}$, where $k T$ and $E M$ are the $\mathrm{X}$-ray temperature and emission measure, and IN and OUT define the region within the radius $\approx 0.05 R_{180}$ and the region within the the annulus with bounding radii $0.05-0.20 R_{180}$, respectively. Rossetti \& Molendi (2010) prove that the pseudo-entropy ratio used in their study is a good proxy for the deprojected quantity, and Rossetti et al. (2011, their Fig. 3) find a good correlation between the pseudo-entropy ratio and, for example, the central entropy defined in Cavagnolo et al. (2009). We measured $\mathcal{E}=0.833 \pm 0.097$, which places A2254 among clusters with high entropy core (see also Sect. 5.1).

We also prepared a 2D map of X-ray temperature, starting from the EPIC images. We used a modified version of the adaptive binning and broad band fitting technique described in Rossetti et al. (2007), where we substituted the Cappellari \& Copin (2003) adaptive binning algorithm with the weighted Voronoi tessellation by Diehl \& Statler (2006, see Rossetti \& Molendi 2010). The temperature map is shown in Fig. 23. The formal errors given in output from the procedure range from $15 \%$ 


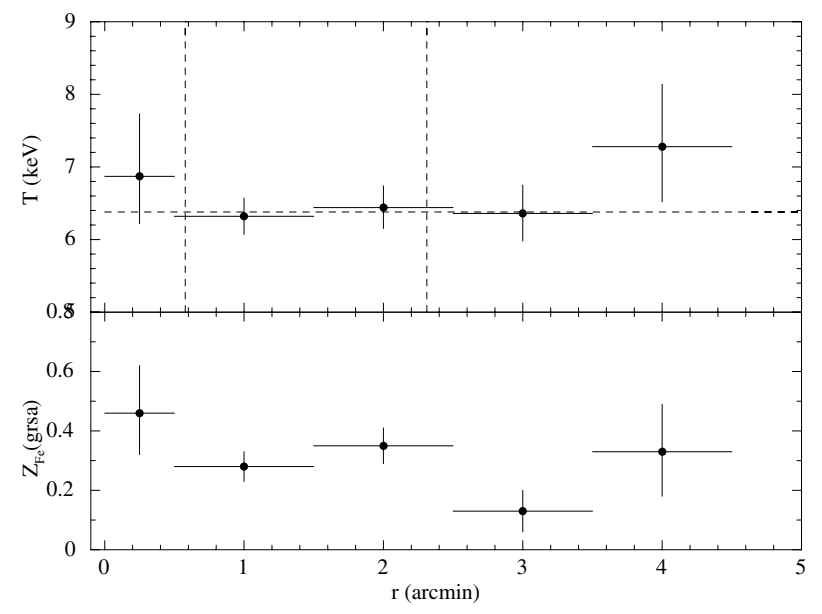

Fig. 22. Profiles of the azimuthally averaged, projected temperature and metal abundances of A2254. The error bars are at the $68 \%$ c.l. The horizontal line shows the value of $k T_{\text {OUT }}$, i.e. the value of $k T$ estimated within the $0.05 R_{180}<r<0.2 R_{180}$ region, with the two radii indicated by the two vertical lines.

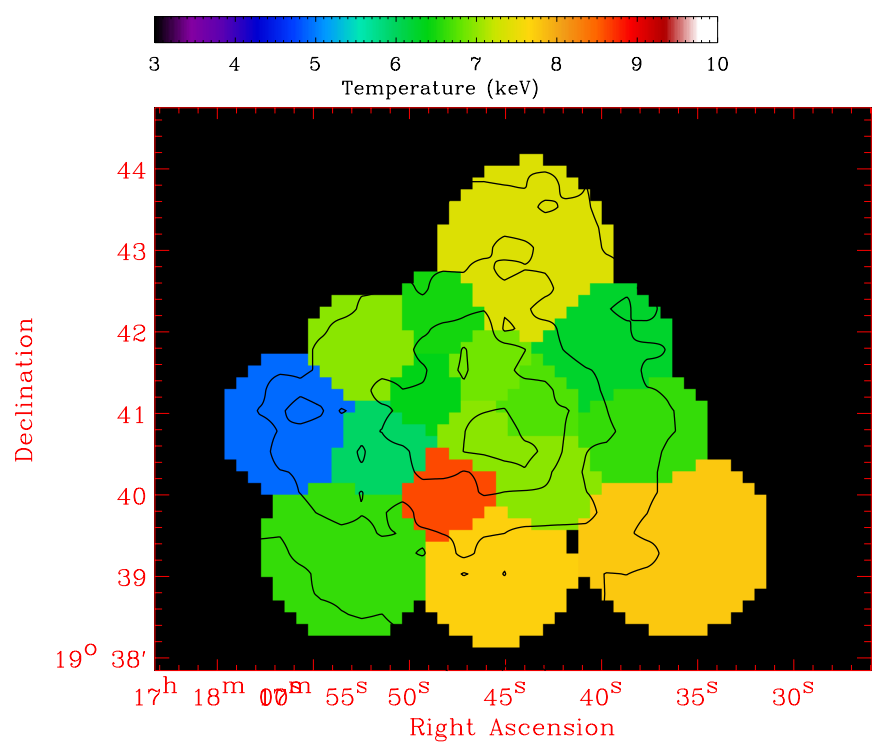

Fig. 23. Temperature map (keV) with, superimposed, the contour levels of the XMM image. Coordinates are right ascension and declination (J2000).

to $20 \%$, but the measures at the map periphery, more affected by the background, should be considered with more caution.

\section{Discussion and conclusions}

\subsection{Cluster structure}

The high values of the velocity dispersion $\sigma_{V}=$ $1340_{-84}^{+101} \mathrm{~km} \mathrm{~s}^{-1}$ and X-ray temperature $k T=(6.38 \pm 0.25) \mathrm{keV}$ are comparable to the values found for hot, massive clusters (Mushotzky \& Scharf 1997; Girardi \& Mezzetti 2001). However, our estimates of $\sigma_{V}$ and $T_{\mathrm{X}}$ are inconsistent at the 3 -sigma level with each other under the assumption of the equipartition of energy density between ICM and galaxies. In fact, we obtain $\beta_{\text {spec }}=1.71_{-0.21}^{+0.26}$ to be compared with $\beta_{\text {spec }}=1$, where $\beta_{\text {spec }}=\sigma_{\mathrm{v}}^{2} /\left(k T / \mu m_{\mathrm{p}}\right)$ with $\mu=0.58$ the mean molecular weight and $m_{\mathrm{p}}$ the proton mass (see also Fig. 6). Evidence that
A2254 is far from the dynamical equilibrium comes from both optical and X-ray analyses, as discussed in the following.

From the optical point of view, we find strong evidence of bimodality from our 2D analysis of the photometric sample with the E and W peaks separated by $\sim 0.4-0.5 h_{70}^{-1} \mathrm{Mpc}$. The (modified) DS-test also shows evidence of substructure at a very high level (at the $99.99 \%$ c.l.). Our 3D analysis of the region of the 2D E peak, detects a high-velocity subcluster, which explains the high velocity-dispersion in that region and the strong increase in the velocity-dispersion profile at $\sim 0.4-0.5 h_{70}^{-1} \mathrm{Mpc}$ from the cluster center (see Fig. 6).

However, a simple bimodal structure is not completely satisfactory when looking at the whole observational scenario. In fact, the W peak is not well centered on the BCG and both E and $\mathrm{W}$ peaks seem to have, in their turn, a bimodal or a northsouth elongated structure (see Figs. 12 and 13). The E subcluster recovered through the $3 \mathrm{D}$ analysis is a minor structure and contains no dominant galaxy, so it seems improbable that it causes the presence of the 2D E peak, which is almost as rich as the $\mathrm{W}$ peak (see Table 2). Indeed, more refined 3D analyses (e.g., the H-tree and the 3D-DEDICA methods) show evidence of a more complex structure. In particular, the $\mathrm{H}$-tree method detects a low-velocity eastern subcluster, which can explain even better the important $2 \mathrm{D} \mathrm{E}$ peak and the strong increase in the velocitydispersion profile at $\sim 0.4-0.5 h_{70}^{-1} \mathrm{Mpc}$ from the cluster center. The low-velocity eastern subcluster might also explain the results of the weighted gap analysis (see Fig. 7). The 3D-DEDICA analysis reveals five subclusters, two of which are in the west region: they might explain the presence of a bimodal 2D W peak.

We stress that our identification of subclusters is always confirmed by the 3D-KMM analysis. In principle, one might use the significance (as computed according to the likelihood ratio test statistics) to select the best cluster partition (e.g., Bird 1994). However, we note that in A2254, the high-velocity E group is the only subcluster well contained in the region sampled by our redshift data, while other subclusters have a large size and/or are at the boundary of this region. Thus, although the use of the 3DKMM approach seems useful to perform a homogeneous check of the subclusters detected using other disparate methods, we do not rely on the 3D-KMM results to fix the best partition of subclusters and the relevant kinematical properties. To obtain more stringent conclusions on the structure of A2254 we stress the need for more galaxy redshifts and a more extended sampled region.

As for the X-ray data, all the methods of the morphological analysis provide evidence of a dynamically disturbed cluster: i) the power ratio analysis, with its value of $P_{1}^{\mathrm{pk}} / P_{0}^{\mathrm{pk}}=1.85 \pm$ 0.47 , together with the radio power at $1.4 \mathrm{GHz}$ of $P_{1.4 \mathrm{GHz}}=$ $2.9 \times 10^{24} h_{70}^{-2} \mathrm{~W} \mathrm{~Hz}^{-1}$ (Govoni et al. 2001b) places A2554 in the region of well known radio halo mergers, such as Abell 2256 and Abell 665, on the $P_{1} / P_{0}-P_{1.4 \mathrm{GHz}}$ relation of Buote (2001; see their Fig. 1-left, where $P_{1.4 \mathrm{GHz}}=2.1 \times 10^{24} \mathrm{~W} \mathrm{~Hz}^{-1}$ in their cosmology); ii) the combination of the three morphology indicators (as in Cassano et al. 2010b) in the $(w, c),\left(P_{3} / P_{0}, c\right)$, and $\left(P_{3} / P_{0}, c\right)$ planes designates A2254 as a morphologically distubed cluster among the other clusters with radio halo in the sample of that paper. In fact, A2254 shows higher values of $w$ and $P_{3} / P_{0}$ and a lower value of $c$ with respect to the median values presented in the study of Cassano et al. (2010b, i.e. $w=0.012 \times 500 h_{70}^{-1} \mathrm{kpc}, c=0.2$, and $P_{3} / P_{0}=1.2 \times 10^{-7}$ ) and there used to separate disturbed from relaxed systems; iii) the value of $w=(0.037 \pm 0.001) \times 500 h_{70}^{-1} \mathrm{kpc}$ gives a significant indication of an unrelaxed system. It is an intermediate value among those measured for 45 ROSAT-observed clusters 
by O'Hara et al. (2006). According to the simulations by Poole et al. (2006, their Fig. 11), this value of $w$ is typical of ongoing cluster mergers.

The active dynamical state of A2254 is also confirmed from the thermodinamic point of view, given the absence of a temperature drop in the core and the classification of A2254 as a high-entropy core according to Leccardi et al. (2010), who fix the separation value between the medium and high-entropy core cluster at $\mathcal{E}=0.64(\mathcal{E}=0.833 \pm 0.097$ in A2254). In fact, observations seem to favor cool core destruction through cluster mergers (Allen et al. 2001; Sanderson et al. 2006), and the vast majority of merging systems host high-entropy cores according to Leccardi et al. (2010), who analyzed 59 clusters spanning a wide range of dynamical states. The temperature map, limited by the quality of the data to the inner regions, reveals a hot core with no significant temperature variations, consistent with an ongoing merger.

\subsection{Optical - X-ray - radio data comparison}

On a large scale, when comparing data from different wavelengths, the main feature is the correlation between the elongation of the optical structure, with the two galaxy clumps separared by $\sim 0.5 h_{70}^{-1} \mathrm{Mpc}$ along the east-west direction, and the east-west elongation of the X-ray emission isophotes (see Fig. 1). The elongation of X-ray isophotes in the merging direction is expected from simulations (e.g., Roettiger et al. 1996). On a small scale, the X-ray emission is peaked at the BCG galaxy. Instead, the E peak does not coincide with a secondary X-ray peak. This discrepancy between the distributions of the ICM (collisional) and galaxies (noncollisional component) is a strong point in favor of an ongoing cluster merger.

Our interpretation of the above correlations or noncorrelations in the context of A2254 scenario is the following. The almost "triangular" shape of external X-ray isophotes indicates that the high-velocity E group has just passed through the main system going from west to east, as suggested by the comparison of Fig. 20 with, e.g., the second or third panels - or maybe the fourth panel considering a possible projection effect - in Figs. 4 and 5 of Poole et al. (2006 - top line, where the subcluster comes from the left). This scenario agrees with observing the high-velocity E group of galaxies as more advanced in the merging direction, while the ICM component is slowed down and left behind (see e.g., the bullet cluster by Markevitch et al. 2002). Unlike the bullet cluster, A2254 does not reveal a secondary X-ray peak: this might be due to a more advanced phase of the merger or, in agreement with our mass estimates (see below), with the subcluster being much smaller and thus completely stripped of its gas content.

However, a simple bimodal merger is not completely satisfactory. In fact, the X-ray emission in the internal cluster region, i.e. in the region of the $\mathrm{W}$ peak, also reveals an elongation toward the north, perpendicular to the merging direction (see Figs. 19 and 20). This could be connected to a particular phase of the the east-west merger resembling the high-emission structure perpendicular to the merger at the time of the apocenter (but not in its symmetric aspect, see Fig. 4 of Poole et al. 2006). Alternatively, it could be connected to another accretion phenomenon - along the north-south direction. In fact, optical 2D analyses of deep Subaru data (see Figs. 12 and 13) reveal a north-south double structure in the cluster center: one enclosing the BCG and one slightly north. The 3D DEDICA analysis finds northern and southern galaxy clumps in the west part of the cluster, too.
As for its radio morphology, A2254 is the most irregular in the sample of Govoni et al. (2001b). The halo is not centered on the region of the maximum X-ray emission (see its upsidedown "U" shape in Figs. 1 and 20), in some respects resembling the behavior of radio relics. In the comparison between radio and other data, the main feature is its bad correlation, already on large scales, with X-ray emission, and with the merging direction suggested by the two optical subclusters. In fact, here the halo does not permeate the cluster volume in a similar way to the X-ray emitting gas of the intracluster medium (see Fig. 1), as generally shown in other clusters not only by their large scale morphology but also by the point-to-point correlation between radio and X-ray brightness (see the case of the four clusters analyzed by Govoni et al. 2001a). Moreover, halos are round or elongated in the direction of the merger (the bullet cluster by Markevitch et al. 2002; Abell 520 by Girardi et al. 2008; Abell 754 by Macario et al. 2011): this is not the case for the A2254 halo.

Our interpretation is that the peculiar radio morphology of A2254 likely comes from the very complex substructure of A2254. In particular, the two north-south elongated branches of the radio emission might be connected with the minor northsouth mergers suggested by our optical analysis. Although the "U" shape morphology of the radio halo (see Fig. 1) recalls the " $H$ " shape of the contours in the galaxy distribution in Fig. 12, the two features are shifted with respect to the other along the east-west direction.

Finally, we stress the absence of a connection between bright galaxies and subclusters, while this is often found in complex systems in 2D (e.g., Geller \& Beers 1982), in 3D (e.g., Abell 520, Girardi et al. 2008), and also when a subcluster is partially destroyed (see the beautiful example of the tidal debris in A2744 by Owers et al. 2011). In A2254, the high-velocity E peak, the one that is detected best through our optical analysis, hosts a bright, dominant galaxy. The feature that is worthy of mention, in spite of the small-sample statistics, is that BCG2, BCG3, and BCG4 are all located on the eastern side of the cluster, more external than the E subcluster. However, their optical spectra do not show features - e.g. emission lines or a strong $\mathrm{H}_{\delta}$ absorption - typical of a possible starburst or poststarburst activity triggered by the shocked gas during a cluster-cluster merger (Bekki et al. 2010), thus there is no clear connection with the ongoing merger.

\subsection{A simple model for mass and merging scenario}

Following the above discussion, A2254 can be described in first approximation by a main system and an eastern highvelocity group. Looking at Table 3 we can estimate $\sigma_{V} \sim$ $1000-1200 \mathrm{~km} \mathrm{~s}^{-1}$ and $\sigma_{V}=200-500 \mathrm{~km} \mathrm{~s}^{-1}$ for the main and the E group, respectively.

Making the usual assumptions for the main system and the subcluster (cluster sphericity, dynamical equilibrium, coincidence of the galaxy and mass distributions), we can compute virial global quantities. We follow the prescriptions of Girardi \& Mezzetti (2001; see also Girardi et al. 1998) and compute $R_{\text {vir }}$ an estimate for $R_{200}$ - and the mass contained within this radius. In particular, we assume $R_{\text {vir }}=0.17 \times \sigma_{v} / H(z) h_{70}^{-1} \mathrm{Mpc}$ for the radius of the quasi-virialized region (see Eq. (1) of Girardi \& Mezzetti 2001; with the scaling of $H(z)$ of Eq. (8) of Carlberg et al. 1997, for $\left.R_{200}\right)$. For the mass we use $M=M_{V}-S P T=$ $3 \pi / 2 \cdot \sigma_{V}^{2} R_{\mathrm{PV}} / G-\mathrm{SPT}$ (Eq. (3) of Girardi \& Mezzetti 2001), with $\mathrm{R}_{\mathrm{PV}}$ computed using the full procedure (see also Eq. (13) of Girardi et al. 1998, with $A=R_{\text {vir }}$ for a close approximation) 
and the surface pressure term correction $S P T=0.2 \times M_{V}$. In practice, both $R_{\mathrm{vir}}$ and $M$ can be computed on the basis of the estimated velocity dispersion with the usual scaling laws $R_{\mathrm{vir}} \propto \sigma_{V}$ and $M\left(<R_{\text {vir }}\right) \propto \sigma_{V}^{3}$.

We computed $M\left(<R_{\mathrm{vir}}=2.2-2.7 \quad h_{70}^{-1} \mathrm{Mpc}\right)=1.5-2.5 \times$ $10^{15} h_{70}^{-1} \quad M_{\odot}$ for the main system and $M\left(<R_{\text {vir }}=0.4-\right.$ $\left.1.2 h_{70}^{-1} \mathrm{Mpc}\right)=0.1-1.8 \times 10^{14} h_{70}^{-1} M_{\odot}$ for the E group, leading to a low mass ratio for the merger $(<(1: 10))$. We computed a mass $M_{\text {sys }}\left(<R=2.2-2.7 h_{70}^{-1} \mathrm{Mpc}\right)=1.5-2.9 \times 10^{15} h_{70}^{-1} M_{\odot}$ for the whole system, where we assumed that $M \propto R$ roughly extrapolates the mass of the $\mathrm{E}$ group. According to the virial scaling laws, the above mass range leads to $\sigma_{V} \sim$ (10001250) $\mathrm{km} \mathrm{s}^{-1}$ for the virial velocity dispersion, thus making the $\beta_{\text {spec }}=1$ model acceptable.

For comparison, we would obtain $M_{\mathrm{sys}, 1}\left(<R_{\mathrm{vir}, 1}=\right.$ $\left.3.0 h_{70}^{-1} \mathrm{Mpc}\right)=(3.5 \pm 1.0) \times 10^{15} h_{70}^{-1} M_{\odot}$ when assuming A2254 as a dynamically relaxed cluster and $M_{\text {sys,5 }} \sim$ $4 \times 10^{15} h_{70}^{-1} M_{\odot}$ when assuming A2254 is the very complex cluster formed by the five DEDICA subclusters.

We used X-ray data to compute an alternative mass estimate. Given the insignificant departures from the isothermality of the temperature profile out to $\sim 0.9 h_{70}^{-1} \mathrm{Mpc}$ (see Fig. 22), we derived a mass estimate using the isothermal beta model where the total mass profile can be expressed by a simple analytical formula (e.g., Eq. (6) of Henry et al. 1993, with $\gamma=1$ ). Using the value of $k T_{\text {OUT }}$ and the fitted parameters of the $\beta$ model to the surface brightness (Sect. 4.1), we obtained a mass $M_{\mathrm{X}}\left(<R=0.9 h_{70}^{-1} \mathrm{Mpc}\right)=0.48 \times 10^{15} h_{70}^{-1} M_{\odot}$ with nominal errors less than the $5 \%$. We rescaled the optical mass $M_{\text {sys }}$ to $R=0.9 h_{70}^{-1} \mathrm{Mpc}$ assuming that the system is described by a King-like mass distribution with a very small core radius (Girardi et al. 1998) or, alternatively, an NFW profile where the mass-dependent concentration parameter $c$ is taken from Navarro et al. (1997) and rescaled by the factor $1+z$ (Bullock et al. 2001; Dolag et al. 2004), here $c \sim 4$. We obtain $M_{\text {sys }}(<R=$ $\left.0.9 h_{70}^{-1} \mathrm{Mpc}\right)=(0.6-1.0) \times 10^{15} h_{70}^{-1} M_{\odot}$, to be compared with $M_{\mathrm{X}}\left(<R=0.9 h_{70}^{-1} \mathrm{Mpc}\right) \sim 0.5 \times 10^{15} h_{70}^{-1} M_{\odot}$. Thus, when opportunely correcting the optical mass for the presence of substructure, we do not find a large discrepancy between optical and X-ray mass estimates, in agreement with $\beta_{\mathrm{spec}}=1$ being acceptable (see above).

Taking the face-on values above, one might estimate that the nonthermal pressure in A2254 ranges from a value of $10 \%$ to $100 \%$ of the gas thermal pressure. However, the above comparison of the two mass estimates should at best be considered tentative since the multiclump structure of the cluster should be better known. Indeed, previous efforts at making a comparison to put a limit on nonthermal pressure have targeted systems that can be assumed to be in equilibrium, at least in the noncollisional component (e.g., a sample of elliptical galaxies by Churazov et al. 2010).

We can use our results to examine A2254 with respect to the observed scaling relations among $P_{1.4 \mathrm{GHz}}$, the halo radio power, and $M_{\mathrm{H}}$, the total cluster mass contained within the size of the radio halo, $R_{\mathrm{H}}$ (Cassano et al. 2007). We rescaled the mass $M_{\text {sys }}$ to $R_{\mathrm{H}}=0.4 h_{70}^{-1} \mathrm{Mpc}$ (Cassano et al. 2007) and obtain $M_{\text {sys }}\left(<R_{\mathrm{H}}=0.4 h_{70}^{-1} \mathrm{Mpc}\right)=(0.2-0.5) \times 10^{15} h_{70}^{-1} M_{\odot}$. Thus A2254 agrees with the relation fitted for other clusters with radio halos (see Fig. 9 of Cassano et al. 2007).

We also attempted to analyze the cluster merger between the main system and the high-velocity E group. Even if the substructure of A2254 is more complex, the elongation of

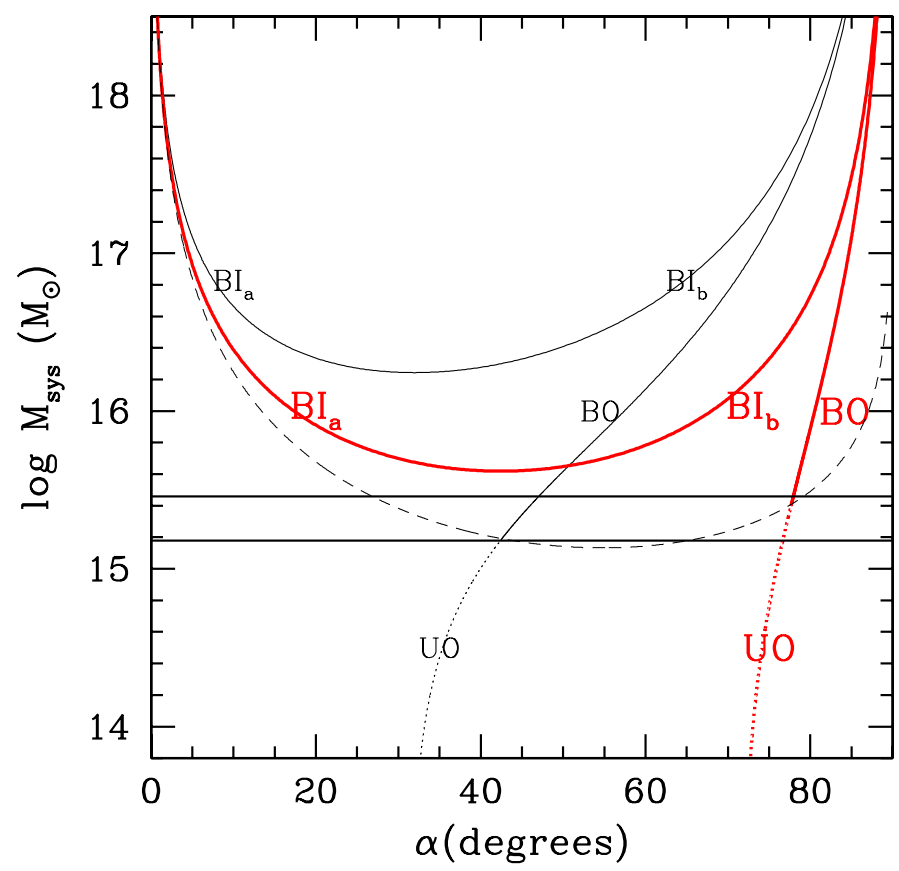

Fig. 24. System mass vs. projection angle for bound and unbound solutions (thick solid and thick dashed curves, respectively) of the twobody model applied to the high-velocity $\mathrm{E}$ group and the main cluster. Thin/black and thick/red lines refer to $t=0.1 \mathrm{Gyr}$ and $t=0.5 \mathrm{Gyr}$, respectively. Labels $\mathrm{BI}_{\mathrm{a}}$ and $\mathrm{BI}_{\mathrm{b}}$ indicate the bound and incoming, i.e., collapsing solutions (solid curve). Labels $\mathrm{BO}$ and $\mathrm{UO}$ indicate the bound outgoing, i.e., expanding solutions and unbound outgoing solutions (solid curve going on in the dotted curve, respectively). The horizontal lines give the range of observational values of the mass system. The thin dashed curve separates bound and unbound regions according to the Newtonian criterion (above and below the thin dashed curve, respectively).

the X-ray surface brightness suggests that this merger is the most recent one. We applied the two-body model (Beers et al. 1982; Thompson 1982) following the methodology outlined for other DARC clusters, e.g., Abell 520 (Girardi et al. 2008) and Abell 2345 (Boschin et al. 2010). The values of the relevant parameters for the two-subcluster system are the above $M_{\text {sys }}=1.5-2.9 \times 10^{15} h_{70}^{-1} M_{\odot}$, the relative LOS velocity in the rest frame, $\Delta V_{\text {rf.LOS }} \sim 3000 \mathrm{~km} \mathrm{~s}^{-1}$, and the projected linear distance between the two clumps, $D \sim 0.5 h_{70}^{-1} \mathrm{Mpc}$, taken as the distance between the BCG and the 2D E peak. The comparison between the observed shape of the X-ray surface brightness isophotes and the simulated ones (Poole et al. 2006) suggests we can assume we see the merger after the core crossing and not too long after the apocenter. For comparison with other clusters hosting radio halos, the time $t$ elapsed from the core crossing is generally few fractions of Gyr (e.g., Markevitch et al. 2002; Girardi et al. 2008). Figure 24 compares the bimodalmodel solutions as a function of $\alpha$, where $\alpha$ is the projection angle between the plane of the sky and the line connecting the centers of the two clumps, with the observed mass of the system $M_{\text {sys }}$. We considered two different cases $(t=0.1 \mathrm{Gyr}$ and $t=0.5 \mathrm{Gyr}$ ), and find a bound outgoing solution (BO) with $\alpha \sim 45^{\circ}$, and $\alpha \sim 75^{\circ}$, respectively. These intermediate angles agree somewhat with we detect the E peak in the 3D analysis, which is the most efficient for intermediate angles (Pinkney et al. 1996). These angles lead to a deprojected relative velocity $\Delta V_{\mathrm{rf}} \sim 3100-4200 \mathrm{~km} \mathrm{~s}^{-1}$ of the subcluster with respect to the 
main system, comparable to that of the bullet cluster (Markevitch et al. 2002) or of Abell 2744 (Boschin et al. 2006).

In conclusion, for its high mass and unrelaxed dynamical status, A2254 fits well among typical clusters with the radio halos described in the literature. Present data agree with the scenario of a recent merger with a minor subcluster, but we have evidence of a more complex dynamical history, which should be investigated more to explain the peculiar radio morphology.

Acknowledgements. We are in debt to Federica Govoni for the VLA radio image and with Daniele Dallacasa, Simona Ghizzardi, and Simona Giacintucci for useful discussions. Philip J. Humphrey is thanked for the use of his surfacebrightness fitting code and D. A. Buote is thanked for the use of his X-ray morphology code and for useful discussions, too. We would like to thank Mariachiara Rossetti for the use of her temperature-map code and for useful discussions. M.G. and F.G. acknowledge financial support from ASI-INAF (I/088/06/0 grant) and ASI-INAF (I/009/10/0 contract). This work has been supported by the Programa Nacional de Astronomía y Astrofísica of the Spanish Ministry of Science and Innovation under grants AYA2010-21322-C03-02, AYA200767965-C03-01, and AYA2010-21887-C04-04. This publication is based on observations made on the island of La Palma with the Italian Telescopio Nazionale Galileo (TNG) and the Isaac Newton Telescope (INT). The TNG is operated by the Fundación Galileo Galilei - INAF (Istituto Nazionale di Astrofisica). The INT is operated by the Isaac Newton Group. Both telescopes are located in the Spanish Observatorio of the Roque de Los Muchachos of the Instituto de Astrofisica de Canarias. This publication is also based on observations obtained with XMM-Newton, an ESA science mission with instruments and contributions directly funded by ESA Member States and NASA. This research made use of the NASA/IPAC Extragalactic Database (NED), which is operated by the Jet Propulsion Laboratory, California Institute of Technology, under contract with the National Aeronautics and Space Administration.

\section{References}

Abell, G. O., Corwin, H. G. Jr., \& Olowin, R. P. 1989, ApJS, 70, 1 Allen, S. W., Ettori, S., \& Fabian, A. C. 2001, MNRAS, 324, 877 Arnaud, M., Pointecouteau, E., \& Pratt, G. W. 2005, A\&A, 441, 893 Ashman, K. M., Bird, C. M., \& Zepf, S. E. 1994, AJ, 108, 2348 Bardelli, S., Zucca, E., Vettolani, G., et al. 1994, MNRAS, 267, 665 Bardelli, S., Pisani, A., Ramella, M., Zucca, E., \& Zamorani, G. 1998, MNRAS, 300, 589

Bardelli, S., Schinnerer, E., Smolčic, V., et al. 2011, A\&A, 511, A1 Barrena, R., Ramella, M., Boschin, W., et al. 2005, A\&A, 444, 685 Barrena, R., Boschin, W., Girardi, M., \& Spolaor, M. 2007, A\&A, 469, 861 Barrena, R., Girardi, M., Boschin, W., \& Dasí, M. 2009, A\&A, 503, 357 Beers, T. C., Geller, M. J., \& Huchra, J. P. 1982, ApJ, 257, 23

Beers, T. C., Flynn, K., \& Gebhardt, K. 1990, AJ, 100, 32

Beers, T. C., Forman, W., Huchra, J. P., Jones, C., \& Gebhardt, K. 1991, AJ, 102, 1581

Beers, T. C., Gebhardt, K., Huchra, J. P., et al. 1992, ApJ, 400, 410

Bekki, K., Owers, M. S., \& Couch, W. J. 2010, ApJ, 718, L27

Bertin, E., \& Arnouts, S. 1996, A\&AS, 117, 393

Bird, C. M. 1994, AJ, 107, 1637

Bird, C. M., \& Beers, T. C. 1993, AJ, 105, 1596

Biviano, A., Katgert, P., Thomas, T., \& Adami, C. 2002, A\&A, 387, 8

Biviano, A., Murante, G., Borgani, S., et al. 2006, A\&A, 456, 23

Bonafede, A., Feretti, L., Giovannini, G., et al. 2009, A\&A, 503, 707

Boschin, W., Girardi, M., Spolaor, M., \& Barrena, R. 2006, A\&A, 449, 461

Boschin, W., Barrena, R., \& Girardi, M. 2010, A\&A, 521, A78

Brunetti, G., Cassano, R., Dolag, K., \& Setti, G. 2009, A\&A, 507, 661

Bullock, J. S., Kolatt, T. S., Sigad, Y., et al. 2001, MNRAS, 321, 559

Buote, D. A. 2001, ApJ, 553, L15

Buote, D. A. 2002, in Merging Processes in Galaxy Clusters, ed. L. Feretti,

I. M. Gioia, \& G. Giovannini, Astrophysics, Space Science Library (The Netherlands: Kluwer Ac. Pub.), 79

Buote, D. A., \& Tsai, J. C. 1996, ApJ, 458, 27

Carlberg, R. G., Yee, H. K. C., \& Ellingson, E. 1997, ApJ, 478, 462

Cappellari, M., \& Copin, Y. 2003, MNRAS, 342, 345

Cassano, R., \& Brunetti, G. 2005, MNRAS, 357, 1313

Cassano, R., Brunetti, G., \& Setti, G. 2006, MNRAS, 369, 1577

Cassano, R., Brunetti, G., Setti, G., Govoni, F., \& Dolag, K. 2007, MNRAS, 378, 1565

Cassano, R., Brunetti, G., Röttgering, H. J. A., \& Brüggen, M. 2010a, A\&A, 509, A68

Cassano, R., Ettori, S., Giacintucci, S., et al. 2010b, ApJ, 721, 82
Cavagnolo, K. W., Donahue, M., Voit, G. M., \& Sun, M. 2009, ApJS, 182, 12 Churazov, E., Tremaine, S., Forman, W., et al. 2010, MNRAS, 404, 1165 Crawford, C. S., Edge, A. C., Fabian, A. C., et al. 1995, MNRAS, 274, 75 Danese, L., De Zotti, C., \& di Tullio, G. 1980, A\&A, 82, 322

den Hartog, R., \& Katgert, P. 1996, MNRAS, 279, 349

Diehl, S., \& Statler, T. S. 2006, MNRAS, 368, 497

Dolag, K., Bartelmann, M., Perrotta, F., et al. 2004, A\&A, 416, 853

Dressler, A., \& Shectman, S. A. 1988, AJ, 95, 985

Durret, F., Laganá, T. F., \& Bertin, E. 2010, A\&A, 517, A94

Ebeling, H., Voges, W., Böhringer, H., et al. 1996, MNRAS, 281, 799

Ellingson, E., \& Yee, H. K. C. 1994, ApJS, 92, 33

Ensslin, T. A., \& Gopal-Krishna 2001, A\&A, 366, 26

Ensslin, T. A., Biermann, P. L., Klein, U., \& Kohle, S. 1998, A\&A, 332, 395

Ensslin, T. A., Pfrommer, C., Miniati, F., \& Subramanian, K. 2011, A\&A, 527, A99

Ettori, S., Gastaldello, F., Leccardi, A., et al. 2010, A\&A, 524, A68

Fadda, D., Girardi, M., Giuricin, G., Mardirossian, F., \& Mezzetti, M. 1996, ApJ, 473,670

Fasano, G., \& Franceschini, A. 1987, MNRAS, 225, 155

Feretti, L. 1999, MPE Report No. 271

Feretti, L. 2002, in The Universe at Low Radio Frequencies, held 30 Nov.-4 Dec. 1999, Pune, India, ed. A. Pramesh Rao, G. Swarup, \& Gopal-Krishna, Proc. IAU Symp., 199, 133

Feretti, L. 2005, X-Ray and Radio Connections, ed. L. O. Sjouwerman, \& K. K. Dyer), Published electronically by NRAO, http://www . aoc.nrao.edu/ events/xraydio, held 3-6 February 2004 in Santa Fe, New Mexico, USA

Feretti, L., Gioia I. M., \& Giovannini, G. 2002, Merging Processes in Galaxy Clusters, ASSL, 272 (The Netherlands: Kluwer Academic Publisher)

Ferrari, C., Maurogordato, S., Cappi, A., \& Benoist C. 2003, A\&A, 399, 813

Ferrari, C., Govoni, F., Schindler, S., Bykov, A. M., \& Rephaeli, Y. 2008, Space Sci. Rev., 134, 93

Gal, R. R., de Carvalho, R. R., Lopes, P. A. A., et al. 2003, AJ, 125, 2064

Gastaldello, F., Buote, D. A., Humphrey, P. J., et al. 2007, ApJ, 662, 923

Geller, M. J., \& Beers, T. C. 1982, PASP, 94, 421

Giovannini, G., \& Feretti, L. 2002, in Merging Processes in Galaxy Clusters, Diffuse Radio Sources and Cluster Mergers, ed. L. Feretti, I. M. Gioia, \& G. Giovannini (The Netherlands: Kluwer Ac. Pub.)

Girardi, M., Fadda, D., Giuricin, G. et al. 1996, ApJ, 457, 61

Girardi, M., Escalera, E., Fadda, D., et al. 1997, ApJ, 482, 11

Girardi, M., Giuricin, G., Mardirossian, F., Mezzetti, M., \& Boschin, W. 1998, ApJ, 505, 74

Giovannini, G., Tordi, M., \& Feretti, L. 1999, New Astron., 4, 141

Girardi, M., Borgani, S., Giuricin, G., Mardirossian, F., \& Mezzetti, F. 2000, ApJ, 530, 62

Girardi, M., \& Mezzetti, M. 2001, ApJ, 548, 79

Girardi, M., \& Biviano, A. 2002, in Optical Analysis of Cluster Mergers, Merging Processes in Galaxy Clusters, ed. L. Feretti, I. M. Gioia, \& G. Giovannini (The Netherlands: Kluwer Ac. Pub.)

Girardi, M., Barrena, R., \& Boschin, W. 2007, Contribution to Tracing Cosmic Evolution with Clusters of Galaxies: Six Years Later conference - http:// www.si.inaf.it/sesto2007/contributions/Girardi.pdf

Girardi, M., Barrena, R., Boschin, W., \& Ellingson, E. 2008, A\&A, 491, 379

Giovannini, G., Bonafede, A., Feretti, L., et al. 2009, A\&A, 507, 1257

Goto, T., Sekiguchi, M., Nichol, R. C., et al. 2002, AJ, 123, 1807

Govoni, F., Ensslin, T. A., Feretti, L., \& Giovannini, G. 2001a, A\&A, 369, 441

Govoni, F., Feretti, L., Giovannini, G., et al. 2001b, A\&A, 376, 803

Grevesse, N., \& Sauval, A. J. 1998, Space Sci. Rev., 85, 161

Gullixson, C. A. 1992, in Astronomical CCD Observing and Reduction techniques, ed. S. B. Howell, ASP Conf. Ser., 23, 130

Henry, J. P., Briel, U. G., \& Nulsen, P. E. J. 1993, A\&A, 271, 413

Hoeft, M., Brüggen, M., \& Yepes, G. 2004, MNRAS, 347, 389

Kalberla, P. M. W., Burton, W. B., Hartmann, D., et al. 2005, A\&A, 440, 775 (LAB Map)

Kempner, J. C., Blanton, E. L., Clarke, T. E., et al. 2004, Proc. of the Conf. The Riddle of Cooling Flows in Galaxies and Clusters of Galaxies, ed. T. H. Reiprich, J. C. Kempner, \& N. Soker [arXiv: astro-ph/0310263]

Kennicutt, R. C. 1992, ApJS, 79, 225

Keshet, U. 2011, MNRAS, submitted [arXiv: 1011.0729]

Kuntz, K. D., \& Snowden, S. L. 2008, A\&A, 478, 575

Landolt, A. U. 1992, AJ, 104, 340

Leccardi, A., \& Molendi, S. 2008, A\&A, 486, 359

Leccardi, A., Rossetti, M., \& Molendi, S. 2010, A\&A, 510, 82

Ledlow, M. J., \& Owen, F. N. 1996, 112, 9

Loeb, A., \& Mao, S. 1994, ApJ, 435, L109

Lu, T., Gilbank, D. G., Balogh, M. L., \& Bognat, A. 2009, MNRAS, 399, 1858

Lubin, L. M., Brunner, R., Metzger, M. R., Postman, M., \& Oke, J. B. 2000, ApJ, 531, 5

Macario, G., Markevitch, M., Giacintucci, S., et al. 2011, ApJ, 728, 82 
Malumuth, E. M., Kriss, G. A., Dixon, W. Van Dyke, Ferguson, H. C., \& Ritchie, C. 1992, AJ, 104, 495

Markevitch, M. 2010, Proceedings at 12th Marcel Grossman Meeting, Paris, July 2009 (updated with 2010 results) [arXiv: 1010.3660]

Markevitch, M., Gonzalez, A. H., David, L., et al. 2002, ApJ, 567, L27

Mohr, J. J., Fabricant, D. G., \& Geller, M. J. 1993, ApJ, 413, 492

Mushotzky, R. F., \& Scharf, C. A. 1997, ApJ, 482, L13

Navarro, J. F., Frenk, C. S., \& White, S. D. M. 1997, ApJ, 490, 493

Nonino, M., Dickinson, M., Rosati, P., et al. 2009, ApJS, 183, 244

O'Hara, T. B., Mohr, J. J., Bialek, J. J., \& Evrard, A. E. 2006, ApJ, 639, 64

Owen, F., Morrison, G., \& Voges, W. 1999, Proceedings of the workshop Diffuse

Thermal and Relativistic Plasma in Galaxy Clusters, ed. H. Böhringer, L. Feretti, \& P. Schuecker, MPE Report 271, 9

Owers, M. S., Randall, S. W., Nulsen, P. E., et al. 2011, ApJ, 728, 27

Pinkney, J., Roettiger, K., Burns, J. O., \& Bird, C. M. 1996, ApJS, 104, 1

Pisani, A. 1993, MNRAS, 265, 706

Pisani, A. 1996, MNRAS, 278, 697

Poole, G. B., Fardal, M. A., Babul, A., et al. 2006, MNRAS, 373, 881

Popesso, P., Biviano, A., Böehringer, H., Romaniello, M., \& Voges, W. 2005, A\&A, 433, 431

Press, W. H., Teukolsky, S. A., Vetterling, W. T., \& Flannery, B. P. 1992, in Numerical Recipes, second edition (Cambridge University Press)

Quintana, H., Carrasco, E. R., \& Reisenegger, A. 2000, AJ, 120, 511

Ramella, M., Boschin, W., Fadda, D., \& Nonino, M. 2001, A\&A, 368, 776

Ramella, M., Biviano, A., Pisani, A., et al. 2007, A\&A, 470, 39

Rizza, E., Morrison, G. E., Owen, F. N., et al. 2003, AJ, 126, 119
Roettiger, K., Burns, J. O., \& Loken, C. 1996, ApJ, 473, 651

Roettiger, K., Loken, C., \& Burns, J. O. 1997, ApJS, 109, 307

Roettiger, K., Burns, J. O., \& Stone, J. M. 1999, ApJ, 518, 603

Rossetti, M., \& Molendi, S. 2010, A\&A, 510, 83

Rossetti, M., Ghizzardi, S., Molendi, S., \& Finoguenov, A. 2007, A\&A, 463, 839

Rossetti, M., Eckert, D., Cavalleri, B. M., et al. 2011 A\&A, 532, A123

Sanderson, A. J. R., Ponman, T. J., \& O'Sullivan, E. 2006, MNRAS, 372, 1496

Santos, J. S., Rosati, P., Tozzi, P., et al. 2008, A\&A, 483, 35

Sarazin, C. L. 2002, in The Physics of Cluster Mergers Merging Processes in Galaxy Clusters, ed. L. Feretti, I. M. Gioia, \& G. Giovannini (The Netherlands: Kluwer Ac. Pub.)

Sarazin, C. L. 2004, J. Korean Astron. Soc., 37, 433

Saxton, R. D., \& Siddiqui, H. 2002, XMM-SOC-PS-TN-43

Schlegel, D. J., Finkbeiner, D. P., \& Davis, M. 1998, ApJ, 500, 525

Schuecker, P., Böhringer, H., Reiprich, T. H., \& Feretti, L. 2001, A\&A, 378, 408

Serna, A., \& Gerbal, D. 1996, A\&A, 309, 65

Smith, J. A., Tucker, D. L., Kent, S., et al. 2002, AJ, 123, 2121

Struble, M. F., \& Rood, H. J. 1987, ApJS, 63, 555

Thompson, L. A. 1982, in Early Evolution of the Universe and the Present Structure, ed. G. O. Abell, \& G. Chincarini (Dordrecht: Reidel), IAU Symp., 104

Tonry, J., \& Davis, M. 1979, ApJ, 84, 1511

Tribble, P. C. 1993, MNRAS, 261, 57

Venturi, T., Giacintucci, S., Macario, G., et al. 2008, A\&A, 484, 327

Wainer, H., \& Schacht, S. 1978, Psychometrika, 43, 203

West, M. J., \& Bothun , G. D. 1990, ApJ, 350, 36 